\title{
dS spaces and brane worlds in exotic string theories
}

\author{
Ralph Blumenhagen, ${ }^{a}$ Max Brinkmann, ${ }^{a}$ Andriana Makridou, ${ }^{a, b}$ Lorenz Schlechter ${ }^{a}$ \\ and Matthias Traube ${ }^{a}$ \\ ${ }^{a}$ Max-Planck-Institut für Physik (Werner-Heisenberg-Institut), \\ Föhringer Ring 6, 80805 München, Germany \\ ${ }^{b}$ Fakultät für Physik, Ludwig-Maximilians-Universität München, \\ Theresienstr. 37, 80333 München, Germany \\ E-mail: blumenha@mpp.mpg.de, mbrinkm@mpp.mpg.de, amakrido@mpp.mpg.de, \\ lschlech@mpp.mpg.de, mtraube@mpp.mpg.de
}

ABSTRACT: We investigate string-phenomenological questions of Hull's exotic superstring theories with Euclidean strings/branes and multiple times. These are known to be plagued by pathologies like the occurrence of ghosts. On the other hand, these theories exhibit de Sitter solutions. Our special focus lies on the question of the coexistence of such de Sitter solutions and ghost-free brane worlds. To this end, the world-sheet CFT description of Euclidean fundamental strings is generalized to include also the open string/D-brane sector. Demanding that in the "observable" gauge theory sector the gauge fields themselves are non-ghosts, a generalization of the dS swampland conjecture is found.

KeYwords: Conformal Field Models in String Theory, D-branes, Superstrings and Heterotic Strings

ARXIV EPRINT: 2002.11746 


\section{Contents}

1 Introduction 1

2 Preliminaries 4

2.1 Fluxed $A d S \times d S$ solutions 4

2.2 Exotic superstring theories $\quad 6$

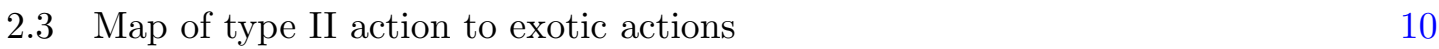

3 Ghosts in exotic string theories 12

3.1 Ghosts for the Lorentzian string 13

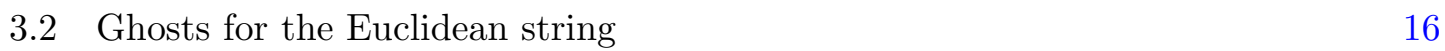

$\begin{array}{lll}3.3 & \text { Speculations and objectives } & 18\end{array}$

4 CFT description of Euclidean exotic strings $\quad 19$

$\begin{array}{lll}4.1 \text { Basics of CFTs with Euclidean world-sheets } & 19\end{array}$

4.2 Boundary states 24

4.3 Fermionic boundary states $\quad 26$

4.4 The influence of space-time signature $\quad 29$

4.5 Orientifolds of Euclidean strings 31

5 D-branes for exotic string theories 33

5.1 D-branes for Euclidean exotic strings 33

5.2 Ghost-free D-brane theories $\quad 37$

5.3 Orientifolds of Lorentzian exotic strings 38

6 Conclusions $\quad 42$

A (A)dS spaces of signature $(p, q) \quad 43$

B Table of branes in exotic IIB theories 44

C Table of branes in exotic IIA theories 45

\section{Introduction}

Even though it is believed that there exists a huge landscape of string compactifications, it turns out to be notoriously difficult to realize certain four-dimensional properties. This led to the idea of the swampland [1] which contains those low-energy effective theories that cannot be UV completed to a consistent theory of quantum gravity. The swampland program intends to extract a set of relatively simple quantitative features that low-energy 
effective field theories should satisfy to admit such an embedding into a theory of quantum gravity (see [2] for a recent review).

In the meantime several such swampland conjectures have been proposed [3-19], triggering further new developments like the emergence proposal [20-22] of infinite distances in field space or the appearance of towers of light strings [23-25]. One of these conjectures is the dS swampland conjecture [8] (see also [26]), whose classical version forbids dS minima altogether, even meta-stable ones with a finite life-time. This conjecture has been refined $[11,12]$ and subsequently related to a quantum argument, the trans-Planckian censorship conjecture [17]. This quantum generalization admits meta-stable dS minima as long as their life-time is sufficiently small.

String theory as we know it is a background dependent formulation of quantum gravity. Therefore, most of the evidence for these conjectures is derived from concrete examples of string compactifications and their effective four-dimensional field theories. It should be mentioned that alternative arguments against de Sitter, based on the concept of quantum breaking [27-30], have been formulated. However, it could well be that the evidence supporting the swampland conjectures is biased by looking just at a certain, well understood, subset of all consistent string theory backgrounds. Can one for instance imagine other backgrounds where de Sitter spaces appear naturally? ${ }^{1}$

Indeed, such settings have been known since the early work of C. Hull et al. [33-36] since 1998. By applying T-dualities along time-like directions, new string theories were proposed. Their common features include that some of the massless fields exhibit the wrong sign in their kinetic terms, and that extra time-like directions can appear. These so-called exotic string theories were shown to form a network related via T- and S-dualities. Roughly one half of these 10D exotic string theories still contain Lorentzian fundamental strings while the other half features Euclidean string world-sheets. In many cases, the open string sector changes to include Euclidean D-branes. ${ }^{2}$ A comprehensive overview of the exotic theories can be found in figure 1. Work towards a perturbative CFT description of Euclidean fundamental strings was started in [39], where it was shown that extra factors of $i=\sqrt{-1}$ appear and need to be taken into account. In such a perturbative approach a number of pathologies arise. One is the aforementioned issue of ghost fields, while another is the appearance of arbitrarily light states upon compactification of time-like directions [39]. It is believed that all these pathologies are rooted in dealing with closed time-like curves, at least in intermediate steps. However, it has been argued [33] that in the UV complete theory these issues could resolve, and the IR pathologies are only a result of the perturbative approach.

Despite these open questions, we think one should not immediately dismiss these models, since their supergravity theories turn out to admit dS solutions. Viewing the presence of ghost fields in the 10D supergravity actions as a feature rather than a bug, it is immediately clear that the standard tree-level dS no-go theorem [40], which was extended in [8],

\footnotetext{
${ }^{1}$ On the same note, in $[31,32]$ the authors argued that time dependent backgrounds might circumvent the dS swampland conjecture.

${ }^{2}$ Questions concerning the backreaction of spherical Euclidean D-branes have been studied lately in a series of papers [37, 38].
} 
does not apply. Indeed, in [33] it was already pointed out that one of these exotic string theories does admit a $d S_{5} \times \mathcal{H}_{5}$ solution, where $\mathcal{H}$ denotes the hyperbolic five-plane. From this more general perspective, one should at least be able to learn something about which physical concepts need to be relaxed to make dS possible.

Moreover, in the past only a few, rather formal aspects of these exotic theories were investigated, so we think it is time to also confront these exotic string theories with more phenomenological questions. For instance, one can ask whether such unconventional theories can nevertheless contain a sector that phenomenologically does resemble our low-energy world. Not much is experimentally known about quantum aspects of gravity, but gauge theories are tested experimentally to very high precision. They are free from physical ghosts and are unitary, or at least any deviation from these principles has escaped detection.

Therefore, in this paper we will start a string-phenomenological study of Hull's exotic string theories. Using complementary methods to the ones employed in [35, 36], we will describe D-branes for Euclidean exotic theories from a formal CFT point of view. This provides the tools to analyze whether such branes can support bona fide gauge theories, though embedded into a closed string background that has some of the strange features already mentioned.

This paper is organized as follows: in a preliminary section 2 we will start by looking at the probably most studied string background, which is the famous type IIB $A d S_{5} \times S^{5}$ space supported by a self-dual five-form background, and observe that in type IIB-like string theories with wrong kinetic terms and/or time-like directions this solution generalizes to $^{3} A d S_{5-m, m} \times d S_{5-n, n}$. This little exercise provides some motivation to contemplate cosmological and phenomenological applications of exotic string theories, which are the natural home for these dS solutions. Next, we will review Hull's exotic string theories and the web of dualities that connects them to ordinary type II string theory.

In section 3 we will discuss the appearance of closed string ghosts and their consequences in more detail. We will also encounter other pathologies that have to do with the appearance of infinitely many ultra-light states once time-like directions are compactified. Such compactifications seem inevitable, if we want to relate the multiple time exotic theories to our $3+1$ dimensional world. While the usual approach of gauging extra world-sheet symmetries (like for the $N=2$ heterotic string [41]) is not an option to get rid of the ghosts, removing (part of) them via an orbifold projection turns out to be feasible.

Continuing the work of [39], in section 4 we develop CFT techniques for the Euclidean exotic string theories and in particular provide the description of the open string sector. Here subtle differences to the standard string theory with Lorentzian world-sheet signature appear, e.g. extra complex phases in the amplitudes. This in turn allows us to constrain the D-brane spectrum of these Euclidean theories by requiring real tensions. We provide a general formula which gives the spectrum of allowed D-branes in any signature.

Finally, in section 5 we will discuss string-phenomenological aspects of these D-brane theories. We complement our results from the previous section with a different construction, employing a mapping motivated by negative tension branes [39] to derive their effective

\footnotetext{
${ }^{3}$ In our notation, signature $(q, p)$ refers to $q$ space-like and $p$ time-like dimensions.
} 
actions. This alternative derivation verifies the brane spectrum found using CFT methods. Both methods agree with the results obtained in [35]. Among the branes we then search for brane-world theories that are phenomenologically viable, i.e. free of massless ghosts and featuring a $(3,1)$ subspace. We will see that while there is such a brane in every exotic theory with Euclidean strings, the O-planes necessary for tadpole cancellation are precisely those from section 3. Therefore, all massless ghosts of the 10D theory, including closed string ghosts, are projected out and the loophole for dS solutions closes. Next we discuss brane worlds for Lorentzian exotic string theories. They do seem to admit a ghost-free massless brane sector. However here the problem of ultralight string modes previously encountered for closed strings in section 3 also applies to open string modes. This means, although the truly massless sector is ghost-free, there are infinitely many arbitrarily light states in the theory.

\section{Preliminaries}

In this section we first recall that in theories with more time-like directions the $A d S_{5} \times$ $S^{5}$ solution of type IIB supergravity generalizes to solutions containing de Sitter spaces (cf. [35]). The natural habitat of these solutions are Hull's exotic string theories that we review in the second part of this section.

\section{1 $\quad$ Fluxed $A d S \times d S$ solutions}

The prototype solution of the type IIB superstring theory with flux is $A d S_{5} \times S^{5}$ with self-dual five-form flux supported on $A d S_{5}$ and $S^{5}$, respectively. Of course this theory has just a single time-like coordinate which is part of the $A d S_{5}$ background. The question that we would like to approach in this section is what happens if more than one of the ten directions of type IIB were time-like, i.e. on a space with signature $(10-p, p)$. For the five-form to still satisfy a self-duality relation, one must have $p$ odd.

The 10D effective (quasi-)action governing the dynamics of the metric and a form field $C_{n-1}$ reads

$$
S \sim M_{s}^{8} \int d^{10} x \sqrt{|G|}\left(e^{-2 \phi} R-\frac{\kappa}{2}\left|F_{n}\right|^{2}\right)
$$

where in the following we will set the dilaton to a constant. This is justified for the actual case of interest, namely the R-R four-form, for which in addition one has to impose the self-duality relation $F_{5}= \pm \star F_{5}$ by hand and change the prefactor of $\left|F_{n}\right|^{2}$ to $\kappa / 4$. Here we have left the sign $\kappa= \pm 1$ of its kinetic term open, where $\kappa=1$ is the usual case. The kinetic term of the n-form $F_{n}$ is defined as

$$
\left|F_{n}\right|^{2}=\frac{1}{n !} G^{i_{1} j_{1}} \ldots G^{i_{n} j_{n}} F_{i_{1} \ldots i_{n}} F_{j_{1} \ldots j_{n}} .
$$

The resulting equation of motion for the metric reads

$$
R_{i j}-\frac{1}{2} g_{i j} R=\frac{\kappa}{2(n-1) !}\left(F_{i k_{2} \ldots k_{n}} F_{j}^{k_{2} \ldots k_{n}}-\frac{1}{2 n} g_{i j} F_{k_{1} \ldots k_{n}} F^{k_{1} \ldots k_{n}}\right)
$$


and for $C_{n-1}$

$$
\partial_{i}\left(\sqrt{|G|} F^{i k_{2} \ldots k_{n}}\right)=0 .
$$

We can write the first relation (2.3) as a matrix equation $\mathbf{R}=\kappa \mathbf{T}$.

Now, we want to consider these equations in a theory with more time-like directions. Generalizing the $A d S_{5} \times S^{5}$ solutions, we make the ansatz

$$
A d S_{5-m, m} \times d S_{5-n, n}, \quad \text { with } \quad m+n=p=\text { odd } .
$$

The description of such multiple times AdS and $d S$ spaces is reviewed in appendix A. For a self-dual five-form flux we can then solve (2.4) simply by choosing $F_{5}$ to satisfy the Bianchi identity $d F_{5}=0$. This is the case for

$$
F_{5}=f E^{1} \wedge \ldots \wedge E^{5}-f \mathcal{E}^{1} \wedge \ldots \wedge \mathcal{E}^{5}
$$

with constant $f$ and with the 5-beins of $A d S_{5-m, m}$ and $d S_{5-n, n}$ as reviewed in appendix A. Choosing the same curvature radius $\alpha$ for the AdS and $d S$ factors, the Ricci scalar vanishes and the left hand side of (2.3) becomes

$$
\mathbf{R}=\left(\begin{array}{cc}
-\frac{4}{\alpha^{2}} \eta^{(m, 5-m)} & 0 \\
0 & \frac{4}{\alpha^{2}} \eta^{(n, 5-n)}
\end{array}\right)
$$

The right hand side then is

$$
\kappa \mathbf{T}=\kappa(-1)^{n}\left(\begin{array}{cc}
-\frac{f^{2}}{4} \eta^{(m, 5-m)} & 0 \\
0 & \frac{f^{2}}{4} \eta^{(n, 5-n)}
\end{array}\right) .
$$

Therefore, for $\alpha=4 / f$ the equations of motion are satisfied if we choose $\kappa=1$ for $n$ even and $\kappa=-1$ for $n$ odd.

Let us mention a few special cases: for $m=1, n=0$ one gets the original $A d S_{5} \times S^{5}$ solution and for $m=0, n=1$ one finds $\mathcal{H}_{5} \times d S_{4,1}$, where $\mathcal{H}_{5}$ denotes the hyperbolic 5 -space. However, the price one has to pay to get this simple solution is that the R-R five-form has the wrong sign of the kinetic term.

We note that all these solutions can also be understood by applying $(m-1, n)$ Wickrotations to the respective coordinates of the original type IIB $A d S_{5} \times S^{5}$ background. From this perspective, in order to keep $F_{5}$ purely real or imaginary, one has to apply either an (even, even) or an (odd, odd) number of Wick-rotations. In the first case, $F_{5}$ remains real, giving the solutions with $n$ even and $\kappa=1$. In the second case however, $F_{5}$ becomes purely imaginary, so the sign of the kinetic term indeed changes and one finds the $n$ odd, $\kappa=-1$ solutions. If for the original type IIB the 5 -form is chosen to be self-dual $\star F_{5}=F_{5}$, the Wick rotation changes this to $\star F_{5}=\kappa F_{5}$. Thus, the sign of the kinetic term of $F_{5}$ and the one in the self-duality relation are related.

We have seen that in type IIB-like supergravities with multiple time directions and possibly wrong signs of the kinetic term for the 5 -five form, $d S$ solutions do exist. Of course, our analysis was only applied to a subsector of the full initial type IIB supergravity action so that one might wonder whether fully consistent supergravity or string theories exist that exhibit precisely those two features. 


\section{$2.2 \quad$ Exotic superstring theories}

Since the early work of Hull $[33,34]$ it is known that string theories with exotic signatures arise from the usual type II theories with $(9,1)$ signature by applying successive T-duality also along time-like directions. This leads to an intricate web of dual theories in ten dimensions of more general signature $(10-p, p)$, whose supergravity actions (quadratic in derivatives) are similar to the type II actions but contain kinetic terms of opposite sign. Despite these apparent ghosts, it was argued that each theory of this duality web represents a different limit of ordinary type II theories, and as a full non-perturbative theory should therefore be intrinsically well-behaved.

However, since these theories are reached via a circle compactification of a time-like direction, they could also all be severely pathological, as at an intermediate step closed time-like curves are encountered that are generally thought to be highly problematic. In the course of this paper we assume that this is not the case and that Hull's exotic theories can make sense.

The de Sitter solutions from the previous subsection will find their natural home in these exotic supergravity theories, meaning that they arise as solutions to the effective theories at leading order in derivatives and at weak string coupling. Therefore, it is this limit that we are most interested in. The perturbative spectrum of the exotic closed string theories which arises via quantization of the corresponding fundamental string was recently worked out in [39]. As expected, the perturbative description of exotic theories carries many pathologies, most prominently ghosts. In this section we review the bouquet of exotic string theories, for more details we refer to the original literature.

The zoo of type $\mathbf{I I}^{\boldsymbol{\alpha} \boldsymbol{\beta}}$ theories. T-duality along a space-like direction exchanges type IIA and IIB string theory. Along a time-like direction, this cannot be the case. For instance, Dirichlet and Neumann boundary conditions of a D-brane are interchanged in the direction that T-duality is applied to. Since regular type II theories only contain Lorentzian D-branes, this means that the T-dual theory can only have Euclidean branes.

Adopting the notation introduced in [39], we label the theories as $\operatorname{IIA}_{(10-p, p)}^{\alpha \beta}$ and $\operatorname{IIB}_{(10-p, p)}^{\alpha \beta}$ with two signs $\alpha, \beta \in\{+,-\}$ and the space-time signature $(10-p, p)$. The first sign indicates whether the theory contains Lorentzian $(+)$ or Euclidean $(-)$ fundamental strings, while the second indicates the same for D1/D2 branes. Here, we will call any even (odd) number of time-like directions Euclidean (Lorentzian). If the signature is omitted we assume $(9,1)$. The usual string theories in this notation are $\mathrm{IIA}^{++}, \mathrm{IIB}^{++}$. We will also use the notation IIA $^{\mathrm{L}}$ and $\mathrm{IIB}^{\mathrm{L}}$ collectively for all theories with Lorentzian fundamental strings and $\mathrm{IIA}^{\mathrm{E}}$ and $\mathrm{IIB}^{\mathrm{E}}$ for the ones with Euclidean strings.

Starting from the usual string theories, time-like T-duality as discussed above leads to Euclidean branes of one dimension less. This means that $\left(\mathrm{IIA}^{++} \leftrightarrow \mathrm{IIB}^{+-}\right)$and $\left(\mathrm{IIB}^{++} \leftrightarrow\right.$ $\mathrm{IIA}^{+-}$) are related by time-like T-duality, just as (IIA $\left.{ }^{++} \leftrightarrow \mathrm{IIB}^{++}\right)$and $\left(\mathrm{IIB}^{+-} \leftrightarrow \mathrm{IIA}^{+-}\right.$) are space-like T-duals.

Now taking the strong coupling limit of the type IIB theories, S-duality acts by exchanging $\mathrm{F} 1 \leftrightarrow \mathrm{D} 1$ and NS5 $\leftrightarrow \mathrm{D} 5$, while D3 is self-dual. Then while $\mathrm{IIB}^{++}$is self-dual, $\mathrm{IIB}^{+-}$has Euclidean D-branes that now get exchanged with the fundamental string and 


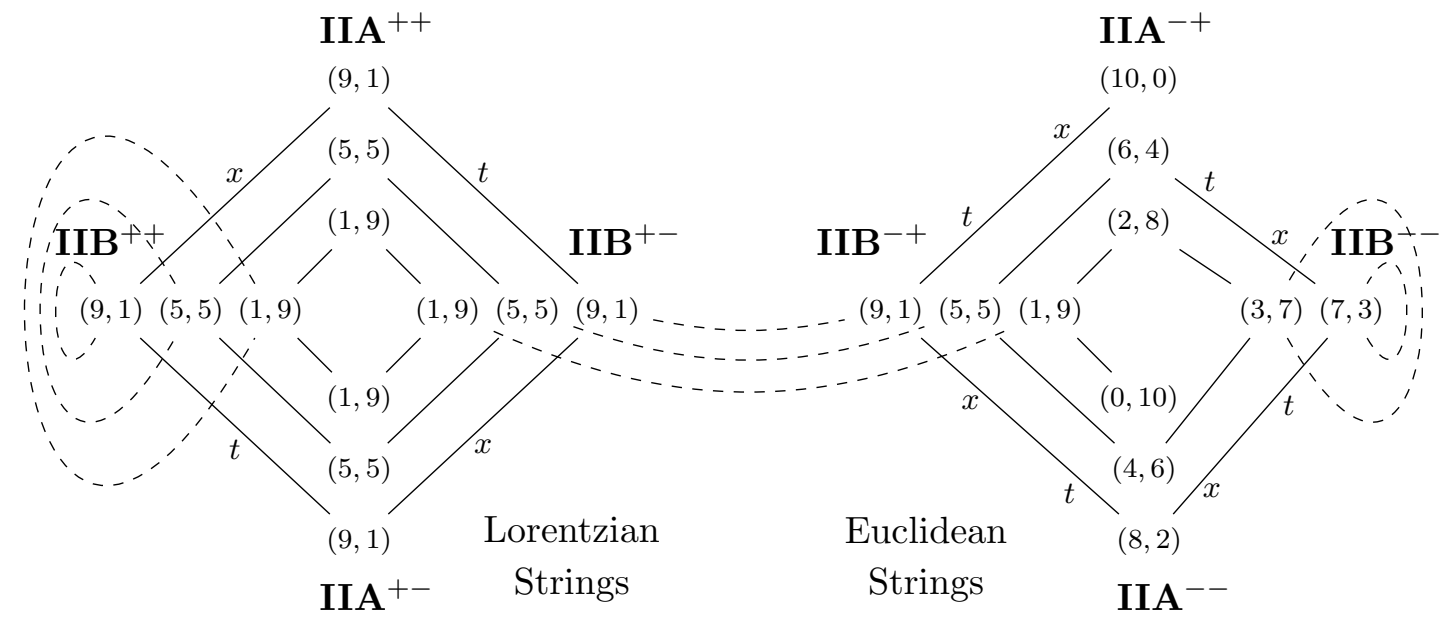

Figure 1. T-dualities (solid lines) and S-dualities (dashed lines) relating type II string theories. The label $x(t)$ indicates dualities arising from compactification on a spatial (time-like) circle. The left side consists of the type $\mathrm{IIA}^{\mathrm{L}} / \mathrm{IIB}^{\mathrm{L}}$ theories with Lorentzian fundamental strings, the theories with Euclidean fundamental strings $\left(\mathrm{IIA}^{\mathrm{E}} / \mathrm{IIB}^{\mathrm{E}}\right.$ ) are on the right. (Diagram adopted from [39]).

NS5-brane. The resulting theory must be of type $\mathrm{IIB}^{-+}$with Lorentzian D1 and D5 and Euclidean F1, NS5 and D3-branes.

One can now complete the type II picture by considering the T-duals of this exotic $\mathrm{IIB}^{\mathrm{E}}$ theory. However since the D-branes of type $\mathrm{IIB}^{-+}$are not homogeneously Lorentzian, one can see that type IIB $^{-+}$compactified along a space-like circle must be dual to a theory compactified along a time-like direction! This theory has Euclidean D2-branes and is thus of type $\operatorname{IIA}_{(8,2)}^{--}$.

All theories with Euclidean F1 have the property that T-duals are with respect to different signature directions. Their respective D-brane spectrum is alternating between Euclidean and Lorentzian as was the case for type $\mathrm{IIB}^{-+}$, and each T-dualization changes the signature. The list of T-dual theories with Euclidean fundamental strings is schematically given by

$$
\operatorname{IIA}_{(10,0)}^{-+} \leftrightarrow \operatorname{IIB}_{(9,1)}^{-+} \leftrightarrow \operatorname{IIA}_{(8,2)}^{--} \leftrightarrow \operatorname{IIB}_{(7,3)}^{--} \leftrightarrow \operatorname{IIA}_{(6,4)}^{-+} \leftrightarrow \ldots \leftrightarrow \operatorname{IIB}_{(1,9)}^{-+} \leftrightarrow \operatorname{IIA}_{(0,10)}^{--}
$$

where going to the right (left) means T-dualizing along a space-like (time-like) direction.

Now that we have found more signatures of type $\mathrm{IIB}^{-+}$, we can S-dualize back to the theories with Lorentzian fundamental strings, where we find that they correspond to type $\mathrm{IIB}^{+-}$with the same signatures. The full bouquet of dual theories and their relations is shown in figure 1.

For completeness let us mention that the strong coupling limit of the type IIA theories are two M-theory variants. In other words, the type IIA theories arise from $\mathrm{M}$ theories with Lorentzian or Euclidean M2-branes on various signatures, compactified on space- or time-like circles. For more on the exotic M-theories see [34, 39]. 
Type $\mathbf{I I}^{\boldsymbol{\alpha} \boldsymbol{\beta}}$ supergravities. The bosonic part of the low-energy SUGRA actions for the exotic theories have been worked out in $[33,34]$. Here we provide the compact presentation given in [39]. As usual the 10D actions are given by a sum over NS-NS, R-R and ChernSimons contributions

$$
S\left[\mathrm{IIA} / \mathrm{B}^{\alpha \beta}\right]=S_{\mathrm{NS}}^{\alpha \beta}+S_{\mathrm{R}}[\mathrm{A} / \mathrm{B}]^{\alpha \beta}+S_{\mathrm{CS}}[\mathrm{A} / \mathrm{B}]
$$

where the NS-NS part is the same for type IIA and IIB and the CS part is independent of $\alpha, \beta$. The respective terms in the action are given by

$$
\begin{aligned}
S_{\mathrm{NS}}^{\alpha \beta} & =\frac{1}{2 \kappa_{10}^{2}} \int \mathrm{d}^{10} x \sqrt{|\operatorname{det} G|} e^{-2 \Phi}\left[\mathcal{R}+4(\nabla \Phi)^{2}-\frac{\alpha}{2}\left|H_{3}\right|^{2}\right], \\
S_{\mathrm{R}}[\mathrm{A}]^{\alpha \beta} & =-\frac{1}{2 \kappa_{10}^{2}} \int \mathrm{d}^{10} x \sqrt{|\operatorname{det} G|}\left[\frac{\alpha \beta}{2}\left|F_{2}\right|^{2}+\frac{\beta}{2}\left|\tilde{F}_{4}\right|^{2}\right], \\
S_{\mathrm{R}}[\mathrm{B}]^{\alpha \beta} & =-\frac{1}{2 \kappa_{10}^{2}} \int \mathrm{d}^{10} x \sqrt{|\operatorname{det} G|}\left[\frac{\alpha \beta}{2}\left|F_{1}\right|^{2}+\frac{\beta}{2}\left|\tilde{F}_{3}\right|^{2}+\frac{\alpha \beta}{4}\left|\tilde{F}_{5}\right|^{2}\right], \\
S_{\mathrm{CS}}[\mathrm{A}] & =-\frac{1}{4 \kappa_{10}^{2}} \int B_{2} \wedge F_{4} \wedge F_{4}, \\
S_{\mathrm{CS}}[\mathrm{B}] & =-\frac{1}{4 \kappa_{10}^{2}} \int B_{2} \wedge F_{3} \wedge F_{5},
\end{aligned}
$$

where $H_{3}=d B_{2}, F_{p}=d C_{p-1}$, and $\tilde{F}_{p}=F_{p}-H_{3} \wedge C_{p-3}$. These actions are all independent of the respective signature $(p, q)$, and as usual one has to additionally require (anti-)selfduality $\tilde{F}_{5}=(\alpha \beta) \star \tilde{F}_{5}$ in the type IIB variants. Note that as in the previous subsection the sign of the kinetic term of the 5 -form flux is correlated with the sign in the self-duality relation. Moreover, we have set the Romans mass of type IIA variants to zero for simplicity.

It is clear that in general these actions feature the appearance of ghost states, i.e. states whose kinetic term has the wrong sign. This sign is the result of two effects. First, there is the overall sign of the kinetic terms in (2.11) and second the combination of signs of the inverse metric factors in

$$
\mathcal{L}_{\text {kin }} \sim-\kappa \sqrt{|G|}\left|F_{n}\right|^{2}=-\frac{\kappa}{n !} \sqrt{|G|} G^{i_{1} j_{1}} \ldots G^{i_{n} j_{n}} F_{i_{1} \ldots i_{n}} F_{j_{1} \ldots j_{n}} .
$$

If $\kappa=+/-1$, an odd/even number of time-like indices indicates a ghost. The presence of ghosts is of course strongly related to the existence of dS solutions. Recall that in section 2 we have seen that theories with ghosts can admit solutions to the SUGRA equations of motion that contain dS factors. These ghosts could arise either due to wrong overall signs of the kinetic terms or due to extra time-like directions. These are precisely the two issues that also appear for the exotic superstring theories, making them the natural framework for a string theory embedding of the dS solutions of section 2 .

It is by now folklore that finding dS vacua in string theory is a highly nontrivial undertaking. As a matter of fact, the dS swampland conjecture [8] explicitly forbids dS vacua in string theory as the scalar potential should always satisfy

$$
|\nabla V|>c V
$$




\begin{tabular}{|lccccc|}
\hline Theory & D0 & D2 & D4 & D6 & D8 \\
\hline $\operatorname{IIA}_{(10,0)}^{-+}$ & $(1,0)$ & - & $(5,0)$ & - & - \\
$\operatorname{IIA}_{(9,1)}^{++}$ & $(0,1)$ & $(2,1)$ & $(4,1)$ & $(6,1)$ & $(8,1)$ \\
$\operatorname{IIA}_{(9,1)}^{+-}$ & $(1,0)$ & $(3,0)$ & $(5,0)$ & $(7,0)$ & $(9,0)$ \\
$\operatorname{IIA}_{(8,2)}^{--}$ & $(0,1)$ & $(3,0),(1,2)$ & $(4,1)$ & $(7,0),(5,2)$ & $(8,1)$ \\
$\operatorname{IIA}_{(6,4)}^{-+}$ & $(1,0)$ & $(2,1),(0,3)$ & $(5,0),(3,2),(1,4)$ & $(6,1),(4,3)$ & $(5,4)$ \\
$\operatorname{IIA}_{(5,5)}^{++}$ & $(0,1)$ & $(2,1),(0,3)$ & $(4,1),(2,3),(0,5)$ & $(4,3),(2,5)$ & $(4,5)$ \\
\hline
\end{tabular}

Table 1. Brane spectrum of IIA ${ }^{\alpha \beta}$ theories.

\begin{tabular}{|lccccc|}
\hline Theory & $\mathrm{D}(-1)$ & $\mathrm{D} 1$ & $\mathrm{D} 3$ & $\mathrm{D} 5$ & $\mathrm{D} 7$ \\
\hline $\operatorname{IIB}_{(9,1)}^{++}$ & - & $(1,1)$ & $(3,1)$ & $(5,1)$ & $(7,1)$ \\
$\operatorname{IIB}_{(9,1)}^{+-}$ & $(0,0)$ & $(2,0)$ & $(4,0)$ & $(6,0)$ & $(8,0)$ \\
$\operatorname{IIB}_{(9,1)}^{-+}$ & $(0,0)$ & $(1,1)$ & $(4,0)$ & $(5,1)$ & $(8,0)$ \\
$\operatorname{IIB}_{(7,3)}^{--}$ & - & $(2,0),(0,2)$ & $(3,1),(1,3)$ & $(6,0),(4,2)$ & $(7,1),(5,3)$ \\
$\operatorname{IIB}_{(5,5)}^{++}$ & - & $(1,1)$ & $(3,1),(1,3)$ & $(5,1),(3,3),(1,5)$ & $(5,3),(3,5)$ \\
$\operatorname{IIB}_{(5,5)}^{+-}$ & $(0,0)$ & $(2,0),(0,2)$ & $(4,0),(2,2),(0,4)$ & $(4,2),(2,4)$ & $(4,4)$ \\
$\operatorname{IIB}_{(5,5)}^{-+}$ & $(0,0)$ & $(1,1)$ & $(4,0),(2,2),(0,4)$ & $(5,1),(3,3),(1,5)$ & $(4,4)$ \\
\hline
\end{tabular}

Table 2. Brane spectrum of $\operatorname{IIB}^{\alpha \beta}$ theories.

where $c$ is an order one number. In fairly general tree-level setups, there exist no-go theorems [40,42] (and extensions in [8]) that explicitly forbid dS vacua. Moreover, in [43] $\mathrm{dS}$ was excluded in parametrically controlled regimes. However, the derivation of the above arguments implicitly assumes that all fields have the usual kinetic terms. The explicit dS solutions show that violations of the dS no-go theorems can potentially arise from the presence of ghost fields. For a concrete set-up, an effective $4 \mathrm{D}$ potential is generated via dimensional reduction of an exotic string theory on some internal space with non-trivial fluxes turned on. Whether this effective potential indeed admits dS minima requires a more detailed investigation, but as long as there are ghosts present we expect that the no-go theorems will not hold.

Type $\mathbf{I I}^{\boldsymbol{\alpha} \boldsymbol{\beta}}$ brane spectrum. The brane spectrum of the exotic superstring theories has been worked out in $[35,36]$ by explicitly solving the equations of motion for the exotic supergravity theories. The results for branes coupling to RR-fields are summarized in tables 1 and 2 in the type $\mathrm{II}^{\alpha \beta}$ notation of [39]. In addition to the branes listed here, in [35] an almost complete spectrum, including NS-NS branes, pp-waves etc., was derived. In the tables an entry $(p, q)$ stands for a $p+q$-dimensional brane with world volume of signature $(p, q)$. Table 1 lists the Dp-branes for all type IIA ${ }^{\alpha \beta}$ theories, while table 2 shows the brane spectrum of the $\operatorname{IIB}^{\alpha \beta}$ theories. 
The "mirror" theories ${ }^{4}$ with reversed signatures are not included in the tables, as their supergravity solutions differ only by an overall minus sign, flipping the world volume signature of the branes. Moreover, for type IIB theories allowing $\mathrm{D}(-1)$-branes the dual space-filling D9-brane is expected to be present. Although not stated in the tables, in [35] it has been argued via T-duality that in $\operatorname{IIA}_{(10,0)}^{-+}$a $\mathrm{D} 8_{(10,0)}^{(9,0)}$ should also exist.

Based on dimensional reduction of super Yang-Mills (SYM) in 10 dimensions, it has been argued in [36] that in the world volume theories of the branes in IIA/B ${ }^{- \pm}$the kinetic term for the gauge field should appear with the opposite sign.

\subsection{Map of type II action to exotic actions}

As has already been observed in [39], by studying negative tension branes and the geometry around them, one can determine a map from the usual type IIA/B actions to all exotic actions of type $\mathrm{IIA}^{\mathrm{E}} / \mathrm{IIB}^{\mathrm{E}}$. Since we will employ this map during the course of this paper, let us review it in more detail.

Such negative branes are extended objects that differ from the ordinary branes by carrying opposite tension and R-R charge. ${ }^{5}$ Studying the black D-brane geometry of a stack of $N_{i}^{+}$Dp-branes and $N_{i}^{-}$negative $\widehat{\mathrm{Dp}}$-branes, one can easily see that there exists an interface, at a finite distance from the stack, where the curvature becomes singular and the harmonic function $H$ appearing in the backreacted geometry vanishes. Within this region $H$ becomes negative. Following [39], one can perform the analytic continuation of the initial background $\mathrm{d} s^{2}=H^{-\frac{1}{2}} \mathrm{~d} s_{p+1}^{2}+H^{\frac{1}{2}} \mathrm{~d} s_{9-p}^{2}$ beyond the interface and get

$$
\begin{aligned}
\mathrm{d} s^{2} & =\omega^{-1} \bar{H}^{-\frac{1}{2}} \mathrm{~d} s_{p+1}^{2}+\omega \bar{H}^{\frac{1}{2}} \mathrm{~d} s_{9-p}^{2} \\
e^{-2 \phi} & =\omega^{p-3} g_{s}^{-2} \bar{H}^{\frac{p-3}{2}} \\
F_{p+2} & =-g_{s}^{-1} \mathrm{~d} \bar{H}^{-1} \wedge \Omega_{p+1}
\end{aligned}
$$

with $\Omega_{p+1}$ denoting the volume form along the branes $\Sigma_{p+1}$ and $g_{s}$ the (asymptotic) string coupling. Moreover, one has $\bar{H} \equiv-H$, with the precise form of $H$ not being relevant for our purposes. What is important here is the factor $\omega= \pm i$, where the sign depends on the way one goes around the singularity. A Weyl transformation is sufficient to get rid of the imaginary factors in the metric:

$$
\mathrm{d} s^{2}=-\bar{H}^{-\frac{1}{2}} \mathrm{~d} s_{p+1}^{2}+\bar{H}^{\frac{1}{2}} \mathrm{~d} s_{9-p}^{2}
$$

It is evident that the directions parallel to the brane pick up a relative minus sign, hence the signature of the space-time dynamically changes from $(9,1)$ to $(10-p, p)$. One could think of this as having two different theories at the two sides of the singular interface: one is a usual string theory and the other is an exotic string theory living in the modified spacetime. Within the latter theory, the signature of the brane changes from $(p, 1)$ to $(1, p)$.

\footnotetext{
${ }^{4}$ See $(2.18)$ in the next section for more details.

${ }^{5}$ Note that negative branes $\widehat{\mathrm{Dp}}$ are distinguished from anti-branes $\overline{\mathrm{Dp}}$. The latter carry opposite R-R charge but still positive tension.
} 
Probe branes, under the requirement that they are BPS, can be used to find the nature of the theory inside the bubble, which depends solely on $p$ :

$$
p \text { even : } \operatorname{IIA}_{(10-p, p)}^{-\left(-\frac{p}{2}\right.}, \quad p \text { odd : } \operatorname{IIB}_{(10-p, p)}^{-\left(-\frac{p-1}{2}\right.} .
$$

One can also perform a slightly different Weyl transformation of (2.14), leading to a metric of opposite overall sign:

$$
\mathrm{d} s^{2}=\bar{H}^{-\frac{1}{2}} \mathrm{~d} s_{p+1}^{2}-\bar{H}^{\frac{1}{2}} \mathrm{~d} s_{9-p}^{2}
$$

Adopting this convention, the directions parallel to the negative brane keep their signature, while the transverse directions pick up a minus sign. The space-time signature now becomes $(p, 10-p)$. This might at first seem like an inconsistency, as the choice of conventions for the signature crossing leads to different theories. However, it is understood that there is some kind of "mirror" 6 symmetry between theories in "mirror" space-times, which is

$$
\operatorname{IIA}_{(10-\mathrm{p}, \mathrm{p})}^{\alpha \beta} \leftrightarrow \operatorname{IIA}_{(\mathrm{p}, 10-\mathrm{p})}^{\alpha(-\beta)}, \quad \operatorname{IIB}_{(10-\mathrm{p}, \mathrm{p})}^{\alpha \beta} \leftrightarrow \operatorname{IIB}_{(\mathrm{p}, 10-\mathrm{p})}^{\alpha \beta}
$$

Now, taking into account the phases $\omega$ in the solution (2.14), one can define a general (off-shell) map between the standard and exotic type II actions. We start by defining the transformation of the vielbein determinant $\operatorname{det} e_{\mu}^{a}=\sqrt{\left|\operatorname{det} G_{(9,1)}\right|}$ in order to avoid branch cut issues later. The determinant receives one factor of $\omega^{-1 / 2}\left(\omega^{+1 / 2}\right)$ for each direction parallel (transverse) to the negative brane. Then mapping the vielbein in an appropriate manner reproduces the metric after singularity crossing (2.14), and finally the dilaton field is redefined to get back to the standard dilaton profile. In this manner, the map can be determined as

$$
\begin{aligned}
\operatorname{det} e_{\mu}^{a} & \rightarrow \omega^{-p} \operatorname{det} e_{\mu}^{a}, \\
e_{\mu}^{a}=\left(e_{\|}^{a}, e_{\perp}^{a}\right) & \rightarrow \omega^{\frac{1}{2}}\left(\omega^{-1} e_{\|}^{a}, e_{\perp}^{a}\right), \\
G_{(9,1)} & \rightarrow \omega G_{(10-p, p)}, \\
e^{-\phi} & \rightarrow \omega^{\frac{p-3}{2}} e^{-\phi}, \\
\left.\left(C_{p+1}\right)\right|_{\Sigma_{p+1}} & \rightarrow-\left.\left(C_{p+1}\right)\right|_{\Sigma_{p+1}} .
\end{aligned}
$$

Note that the vielbein does not map to the vielbein of the new theory directly. Indeed, the metric of signature $(10-p, p)$ can be written as

$$
G_{(10-p, p)}=\left(\omega^{-1} e_{\|}^{a}, e_{\perp}^{a}\right) \eta_{(9,1)}\left(\omega^{-1} e_{\|}^{a}, e_{\perp}^{a}\right)^{\top}=\left(e_{\|}^{a}, e_{\perp}^{a}\right) \eta_{(10-p, p)}\left(e_{\|}^{a}, e_{\perp}^{a}\right)^{\top} .
$$

Taking into account that the original metric is

$$
G_{(9,1)}=\left(e_{\|}^{a}, e_{\perp}^{a}\right) \eta_{(9,1)}\left(e_{\|}^{a}, e_{\perp}^{a}\right)^{\top}
$$

\footnotetext{
${ }^{6}$ The term "mirror" here refers to mirroring the signature of the theories (exchanging time and space), not to the usual mirror symmetry of Calabi-Yau manifolds.
} 
we first realize that the second and the third line in (2.19) are compatible, i.e. the transformation of the vielbein implies $G_{(9,1)} \rightarrow \omega G_{(10-p, p)}$. Note that in the two expressions (2.20) and (2.21) for the metrics exactly the same vielbein $e_{\mu}^{a}$ appears so that also the measures are both given by

$$
\sqrt{\left|G_{(9,1)}\right|}=\operatorname{det} e_{\mu}^{a}=\sqrt{\left|G_{(10-p, p)}\right|} .
$$

Then we can avoid branch cuts in the measure factor, ${ }^{7}$ since the measure must map in the same way as the vielbein determinant

$$
\sqrt{\left|G_{(9,1)}\right|} \rightarrow \omega^{-p} \sqrt{\left|G_{(10-p, p)}\right|} .
$$

These maps can be shown to map the supergravity actions according to

$$
\mathrm{IIB} \rightarrow \operatorname{IIB}_{(10-p, p)}^{-(-)^{\frac{p-1}{2}}}, \quad \mathrm{IIA} \rightarrow \operatorname{IIA}_{(10-p, p)}^{-(-)^{\frac{p}{2}}} .
$$

The analysis so far concerns the closed string actions. It is one of the objectives of this paper to generalize this analysis to the open string sector and the corresponding DBI actions on the D-brane world-volumes. Before we move to these questions in section 5, we need to work out a couple of general aspects of ghosts in the exotic string theories.

\section{Ghosts in exotic string theories}

In this section we continue the discussion of ghosts in the exotic string theories. Ghosts are states of negative norm in the Hilbert space, preventing a probabilistic interpretation and, even when removed by hand from the set of physical states, leading to a violation of unitarity. While they might be an important ingredient to find dS solutions, massless or light ghosts are phenomenologically excluded.

A standard procedure to get rid of such states is to gauge more symmetries on the world-sheet, hence introducing new $(b, c)$ ghost systems that change the critical central charge of the theory and cancel the contributions of the problematic ghosts. A famous example is the $N=2$ superstring with a critical central charge of $c=6$ and a fourdimensional target-space of signature $(2,2)$ or $(0,4)[44]$. Due to the extra gauge symmetry, more target-space directions can be gauged away.

However, since we do not want to change the critical dimension, there will only be the usual gauge invariances leading to a critical central charge of 26 (15) for the bosonic (super) string theory. This means there will be a single distinctive time direction and the corresponding $b c$ (and $\beta \gamma$ ) ghost system. Let us analyze the appearance of ghosts for the Lorentzian IIA ${ }^{\mathrm{L}} / \mathrm{IIB}^{\mathrm{L}}$ theories and the Euclidean $\mathrm{IIA}^{\mathrm{E}} / \mathrm{IIB}^{\mathrm{E}}$ theories in more detail. For simplicity, we present the string world-sheet arguments only for the bosonic string, while they analogously hold for the superstring theories.

\footnotetext{
${ }^{7}$ Had we defined the map on the vielbein or the metric instead of $\operatorname{det} e_{\mu}^{a}$ as first principle, the measure would map to something like $\sqrt{-\operatorname{det}\left(\omega G_{(10-p, p)}\right)}$, which in principle gives the same factor $\sqrt{\omega^{-2 p}}$ but now appropriate branch cuts have to be chosen.
} 


\subsection{Ghosts for the Lorentzian string}

The Lorentzian fundamental strings in the $\mathrm{IIA}^{\mathrm{L}} / \mathrm{IIB}^{\mathrm{L}}$ theories can be quantized in complete analogy to the usual IIA and IIB (super-) strings with signature $(9,1)$. This means that the mode algebra for the bosonic fields $X^{\mu}$ reads

$$
\left[\alpha_{m}^{\mu}, \alpha_{n}^{\nu}\right]=m \eta_{(10-p, p)}^{\mu \nu} \delta_{m,-n}
$$

where $\eta_{(10-p, p)}^{\mu \nu}$ denotes the flat metric of signature $(10-p, p) \in\{(9,1),(5,5),(1,9)\}$. In the following let us denote the space-like directions and the single universal time direction with indices $m, n, \ldots$ and the additional new time-like directions by $a, b, \ldots$. Note that the universal time and one space direction can be gauged away as usual. Then for instance the off-diagonal graviton states

$$
\left|V_{G}^{s t}(0)\right\rangle=\epsilon_{\mu a} \alpha_{-1}^{m} \tilde{\alpha}_{-1}^{a}|0\rangle
$$

have negative norm (for $\langle 0 \mid 0\rangle=1$ ) and give physical ghosts that cannot be gauged away. Note that the graviton modes $\left|V_{G}^{s s}(0)\right\rangle=\epsilon_{\mu \nu} \alpha_{-1}^{m} \tilde{\alpha}_{-1}^{n}|0\rangle$ and $\left|V_{G}^{t t}(0)\right\rangle=\epsilon_{a b} \alpha_{-1}^{a} \tilde{\alpha}_{-1}^{b}|0\rangle$ have positive norm.

In the NS-NS sector of the superstring, one only has to replace the $X^{\mu}$ by their fermionic superpartners $\psi^{\mu}$ and the logic goes through analogously. In the R-R sector, there is the distinction between the IIA $/ \mathrm{B}^{++}$and the IIA/ $\mathrm{B}^{+-}$theories, where the latter carry a wrong overall sign for the kinetic terms of the massless R-R fields. This can be taken care of in the world-sheet theory by flipping by hand the overlap between the R-R ground states

$$
\langle 0 \mid 0\rangle_{\mathrm{RR}}^{+-}=-\langle 0 \mid 0\rangle_{\mathrm{RR}}^{++}
$$

Light cone gauge and Lorentz symmetry. The (time-like) T-duality arguments suggest that a change of the target-space signature does not affect the critical dimension of the string theory. Let us check this explicitly on the world-sheet. This can be readily seen for the bosonic string in light cone gauge by checking for anomalies of the $\mathrm{SO}(p, q)$ Lorentz symmetry. The world-sheet metric is fixed as $h_{\alpha \beta}=\eta_{\alpha \beta}$ and we introduce light cone coordinates in space time $X^{+}=1 / \sqrt{2}\left(X^{0}+X^{1}\right), X^{-}=1 / \sqrt{2}\left(X^{0}-X^{1}\right)$, where we singled out one time and spatial direction $X^{0}, X^{1}$. The target-space metric becomes $\eta_{+-}=\eta_{-+}=-1$ for the light cone, $\eta_{a b}=-\delta_{a b}$ for $a, b=1, \ldots, p-1$ remaining time directions and $\eta_{m n}=\delta_{m n}$ for the $m, n=1, \ldots, q-1$ spatial directions.

We follow the standard procedure and look at the open string with (NN) boundary conditions. The remaining gauge freedom is fixed by setting $X^{+}(\sigma, \tau)=x^{+}+p^{+} \tau$. Using the Virasoro constraint equation $\eta_{\mu \nu}\left(\dot{X}^{\mu} \pm X^{\prime \mu}\right)\left(\dot{X}^{\nu} \pm X^{\prime \nu}\right)=0$ to express the oscillator modes of $X^{-}$in terms of the transverse modes yields

$$
\alpha_{n}^{-}=\frac{1}{p^{+}}\left(\frac{1}{2} \sum_{k=-\infty}^{\infty}: \eta_{i j} \alpha_{n-k}^{i} \alpha_{k}^{j}:-a \delta_{n, 0}\right)
$$


with $i, j$ running over the transverse directions and for simplicity setting $\alpha^{\prime}=1 / 2$. The modes still satisfy a "transverse" Virasoro algebra

$$
\begin{aligned}
{\left[p^{+} \alpha_{m}^{-}, p^{+} \alpha_{n}^{-}\right]=} & (m-n) p^{+} \alpha_{m+n}^{-}+ \\
& \left(\frac{D-2}{12}\left(m^{3}-m\right)+2 a m\right) \delta_{m+n}
\end{aligned}
$$

and have commutation relations with the transverse oscillator modes

$$
\left[\alpha_{n}^{i}, p^{+} \alpha_{k}^{-}\right]=n \alpha_{n+k}^{i} .
$$

The only relevant appearance of the space-time metric is in commutation relations $\left[\alpha_{m}^{\mu}, \alpha_{n}^{\nu}\right]$ $=k \eta^{\mu \nu} \delta_{m+n, 0}$. We can use these commutation relations and follow the standard computation for the potentially anomalous commutator $\left[J^{i-}, J^{j-}\right]$ of Lorentz generators $J^{\mu \nu}$. Doing so we find

$$
\left[J^{i-}, J^{j-}\right]=0 \quad \Leftrightarrow \quad D=26, a=1
$$

but no additional constraints on the number of time respectively spatial dimensions. Hence Lorentz symmetry $\mathrm{SO}(p, q)$ is preserved for a total of $p+q=26$ space-time dimensions.

Orbifolding ghosts. Generally, having a theory that has too many degrees of freedom one can proceed in two ways. Either one gauges extra symmetries or one projects out the unwanted states. Since gauging symmetries completely removes the time-like directions, we want to take the second route. Can one remove the massless ghosts by performing an appropriate orbifold projection? In contrast to the gauging procedure an orbifold will not change the critical central charge, but will potentially break the 10D diffeomorphism symmetry to a subgroup.

Following the usual recipe for performing an orbifold in string theory, the untwisted sector is projected to invariant states and a twisted sector must be introduced. Let us discuss appropriate orbifold projections to remove light ghosts from the Lorentzian theories. In the IIA/B $\mathrm{B}_{(9,1)}^{+-}$theory, the ghost R-R fields can be projected out by performing an orbifold by $(-1)^{F_{L}}$. To avoid the appearance of new massless ghosts in the $\mathbb{Z}_{2}$ twisted sector, one can combine this action with a half-shift $S: X \rightarrow X+\pi R$ along a compactified spatial direction. For the IIA $/ \mathrm{B}_{(5,5)}^{++}$theories physical ghosts are related to four extra time-like directions. These ghosts can be removed by taking the quotient by a $\mathbb{Z}_{2}$ reflection $I_{4}: x^{a} \rightarrow-x^{a}$ along these four directions. Similarly, the ghosts in IIA $/ \mathrm{B}_{(1,9)}^{++}$are removed by $I_{8}$, reflecting the eight extra time-like coordinates. Finally, the massless ghosts of IIA $/ \mathrm{B}_{(5,5)}^{+-}$and IIA/B $\mathrm{B}_{(1,9)}^{+-}$ are projected out by $I_{4}(-1)^{F_{L}}$ and $I_{8}(-1)^{F_{L}}$, combining the previous reasoning. These results are summarized in figure 2 .

Compact time-like dimensions. Eventually, we are interested in compactifications of the exotic string theories to 4 dimensions with signature $(3,1)$, so in theories with multiple time-like directions some of them will need to be compactified. The standard problem of compact time-like dimensions are closed time-like curves which violate causality. Since the 


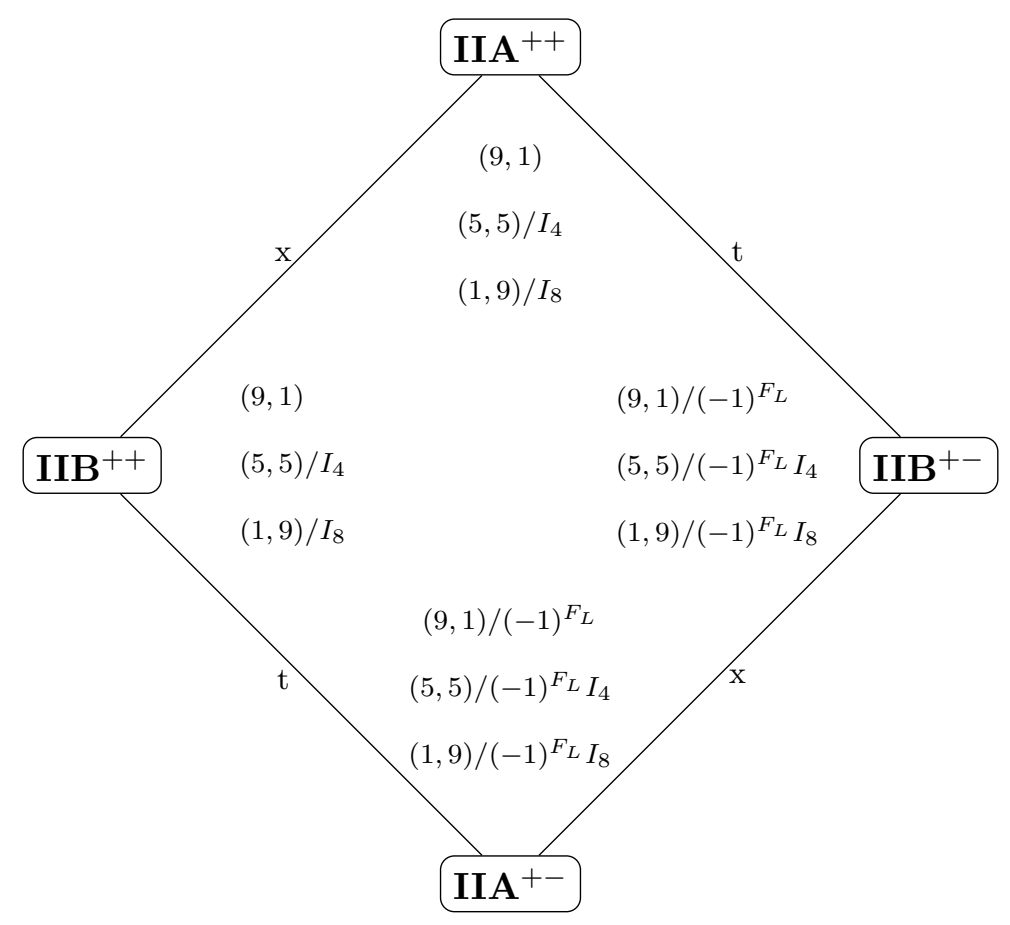

Figure 2. Orbifold projections that remove the massless ghosts for Lorentzian theories. New ghosts in twisted sectors can be avoided by combining these actions with a shift along a spatial direction.

orbifolds project out massless excitations in these directions, one might naively think that the quotient theories are safe. However, we will see that compact time dimensions in exotic string theories lead to further complications.

For the IIA/B $\mathrm{B}_{(9,1)}^{+-}$theory the orbifold by $(-1)^{F_{L}}$ removes all ghost fields from the untwisted sector. In case of the IIA/B $\mathrm{B}_{(5,5)}^{++}$theories however, even though the massless mixed graviton modes $\left|V_{G}^{(\mathrm{ev}, \mathrm{odd})}(0)\right\rangle=\epsilon_{m a} \alpha_{-1}^{m} \tilde{\alpha}_{-1}^{a}|0\rangle$ are projected out, for non-vanishing momentum/energy the linear combination

$$
\left|V_{G}^{(\mathrm{ev}, \mathrm{odd})}\left(p^{n}, e^{b}\right)\right\rangle=\epsilon_{m a} \alpha_{-1}^{m} \tilde{\alpha}_{-1}^{a}\left|p^{n}, e^{b}\right\rangle-\epsilon_{m a} \alpha_{-1}^{m} \tilde{\alpha}_{-1}^{a}\left|p^{n},-e^{b}\right\rangle
$$

remains in the spectrum. Here the upper index pair indicates the behavior of the left and right moving part under the $\mathbb{Z}_{2}$ operation. Here $p^{n}, m, n \in\{0,1, \ldots, 5\}$ denote the usual energy $p^{0}=E$ and space-like momenta, and $e^{b}, a, b \in\{6, \ldots, 9\}$ denote the extra time-like energies. Therefore, there are still massive ghosts in the string spectrum, a $\mathbb{Z}_{2}$ projection does not allow to remove all of them.

The on-shell condition for such a state is

$$
E^{2}+\sum_{a}\left(e^{a}\right)^{2}=\sum_{i}\left(p^{i}\right)^{2}+m^{2}
$$

where $m$ is the mass of the state. We interpret this condition such that for a state of mass $m$ with momenta $p^{i}$ the total energy can be distributed among all the time-like energies such that this quadratic relation is satisfied [45]. Only $E$ is the energy that we have access 
to. Note that while for negative $E$ we have an interpretation in terms of anti-particles with positive $E$, the additional energies $e^{a}$ can be both positive and negative.

Let us now consider a Lorentzian string on a time-like torus of radius $R$. As for a space-like compactification, the time-like momentum (i.e. energy) gets quantized along the compact direction and leads to a mass contribution, resulting in a KK tower of massive states. Similarly, the winding modes contribute to the mass so that in total we find the on-shell condition

$$
E^{2}+\sum_{b}\left[\left(\frac{m_{b}}{R}\right)^{2}+\left(\frac{n_{b} R}{\alpha^{\prime}}\right)^{2}\right]=\sum_{i}\left(p^{i}\right)^{2}+\frac{2}{\alpha^{\prime}}(N+\bar{N}-2 a)
$$

with $a=1 / 2$ for the superstring and the level-matching condition $\sum_{a} m_{a} n_{a}=-(N-\bar{N})$. For $R>\sqrt{\alpha^{\prime}}$ it is tempting to identify a UV cutoff with the Kaluza-Klein scale $\Lambda_{\mathrm{UV}}=1 / R$ that we assume to be only a few orders of magnitude below the string scale. Let us analyze this on-shell condition in the IR regime $|p|<\Lambda_{\mathrm{UV}}$.

In the massless sector $N=\bar{N}=1 / 2$, a non-vanishing time-like $\mathrm{KK} /$ winding mode $\left(m_{a}, n_{a}\right) \neq(0,0)$ already lies outside the IR regime. Thus all the light on-shell states that we have access to are frozen in the extra time directions and feature $e^{a}=0$. Then together with the $\mathbb{Z}_{2}$ projections there are no light ghosts left, so it seems that we are safe. However, for the tower of massive string excitations $N=\bar{N}>1 / 2$ their contribution to the right hand side of (3.10) can be balanced against KK/winding contributions. Therefore, these massive excitations combine with time-like KK/winding modes to appear as extremely light particles from a $4 \mathrm{D}$ perspective. As already observed in [39], even for irrational values of the radius there will always be integers $N, \bar{N}, m_{a}, n_{a}$ such that their $4 \mathrm{D}$ mass lies below any cut-off. Relatedly, there exist kinematically allowed scattering processes like

$$
\begin{gathered}
\left|V_{m_{1}=0}\left(p_{1}^{m}, e_{1}^{a}=0\right)\right\rangle+\left|V_{m_{2}=0}\left(p_{2}^{m}, e_{2}^{a}=0\right)\right\rangle \\
\longrightarrow\left|V_{m_{3}>0}\left(p_{3}^{m}, e_{f}^{a}\right)\right\rangle+\left|V_{m_{4}>0}\left(p_{4}^{m},-e_{f}^{a}\right)\right\rangle
\end{gathered}
$$

with the extra energies in the final state $e_{f}^{a} \neq 0$. Thus, the ultralight states with $N=\bar{N}>$ $1 / 2$ do not decouple in the scattering amplitudes of massless states with $N=\bar{N}=1 / 2$. We can summarize these findings by saying that the dimensionally reduced 10D Lorentzian supergravity actions cannot be considered as Wilsonian effective actions of a $4 \mathrm{D}$ theory.

\subsection{Ghosts for the Euclidean string}

The quantization of the Euclidean fundamental string has been investigated in [39] and features a couple of new aspects and pathologies. Note that this theory is different from the Wick rotated Lorentzian string. In section 4 we will review and continue this analysis, where our special focus will be on the construction of boundary states, providing the CFT description of D-branes for these exotic string theories.

One new aspect of the quantization is that factors of $i=\sqrt{-1}$ appear at various places. For instance, the mode algebra for the bosonic fields $X^{\mu}$ now reads

$$
\left[\hat{\alpha}_{m}^{\mu}, \hat{\alpha}_{n}^{\nu}\right]=-i m \eta_{(10-p, p)}^{\mu \nu} \delta_{m,-n}
$$


As a consequence, the diagonal graviton/B-field states $\left|V_{G}^{s s}(0)\right\rangle$ and $\left|V_{G}^{t t}(0)\right\rangle$ have negative norm and the off-diagonal ones $\left|V_{G}^{s t}(0)\right\rangle$ positive norm (for $\langle 0 \mid 0\rangle=1$ ). However, this is not consistent with the normalization of the Einstein-Hilbert term for the Euclidean string SUGRA actions (2.11). This can be remedied by choosing the correct normalization of the vertex operators. These have been worked out in [39]. The graviton gets an extra factor of $-i$, rendering its norm positive, while the B-field remains a ghost. Of course the time-like ghosts from the previous section also remain in the spectrum.

Orientifolding ghosts. Now we investigate whether there also exist $\mathbb{Z}_{2}$ operations that can mod out all the massless ghost fields for the Euclidean exotic string theories IIA ${ }^{E} / \mathrm{IIB}^{E}$. Let us start with the $\operatorname{IIB}_{(9,1)}^{-+}$theory, which is the S-dual of the $\operatorname{IIB}_{(9,1)}^{+-}$theory. By looking at its SUGRA action (2.11) we see that $H_{3}, F_{1}, F_{5}$ have the wrong sign of the kinetic terms and $F_{3}$ the usual sign. These are precisely the p-form fields that are odd and even under the world-sheet parity transformation $\Omega$, and indeed the S-dual of $(-1)^{F_{L}}$ is known to be $\Omega$. Therefore, the orientifold $\operatorname{IIB}_{(9,1)}^{-+} / \Omega$ has no ghost fields in the closed string sector. Depending on whether the orientifold projection has fixed loci or acts freely (after combining it again with a shift symmetry), there will be a twisted sector in the form of appropriate D-branes that need to be introduced to cancel the R-R tadpole of the O-plane. This open string sector can host additional ghosts. We will come to this point in section 5 .

Now by successively applying spatial T-dualities we can find the orientifold projections for all the IIA $/ \mathrm{B}_{(10-p, p)}^{-, \beta}$ theories. After one T-duality one gets $\operatorname{IIA}_{(8,2)}^{--}$with the projection $\Omega I_{1}$, where $I_{1}$ reflects the new additional time-like coordinate. The corresponding branes are D8-branes localized at a point in the new time-like direction. Another T-duality leads to the $\operatorname{IIB}_{(7,3)}^{--} / \Omega I_{2}(-1)^{F_{L}}$ orientifold, etc. All the resulting quotients are shown in the right hand part of figure 3. T-dualizing instead along the time-like direction, we find the appropriate orientifold quotient to be $\operatorname{IIA}_{(10,0)}^{-+} / \Omega \tilde{I}_{1}(-1)^{F_{L}}$, where $\tilde{I}_{1}$ is a reflection along the space-like direction that was created by T-dualizing.

Compact dimensions. Another new aspect of the Euclidean theories is that the tower of string excitations has imaginary squared mass $m^{2}$. Moreover, since under T-duality a space-like circle maps to a time-like one and vice versa, the winding modes contribute with the opposite sign as the KK modes. Thus, the on-shell relation for a compactification on a torus $T^{D}$ of radii $R_{k}$ with metric $\eta_{k}= \pm 1$ now reads

$$
E^{2}-\sum_{k=1}^{D} \eta_{k}\left[\left(\frac{m_{k}}{R_{k}}\right)^{2}-\left(\frac{n_{k} R_{k}}{\alpha^{\prime}}\right)^{2}\right]=\sum_{i}\left(p^{i}\right)^{2}-i \frac{2}{\alpha^{\prime}}(N+\bar{N}-2 a) .
$$

with the level-matching condition $\sum_{k} \eta_{k} m_{k} n_{k}=N-\bar{N}$. In contrast to the Lorentzian string, here the KK/winding modes can never cancel against the string oscillations. However, for both space-like and time-like compactifications the KK mode contribution can cancel up to arbitrary precision against the winding mode contribution, leading again to the conceptual problem of interpreting the dimensional reduction of the 10D Euclidean supergravity actions as Wilson effective theories. As for the Lorentzian string, these ultralight modes do not decouple in string scattering amplitudes. 


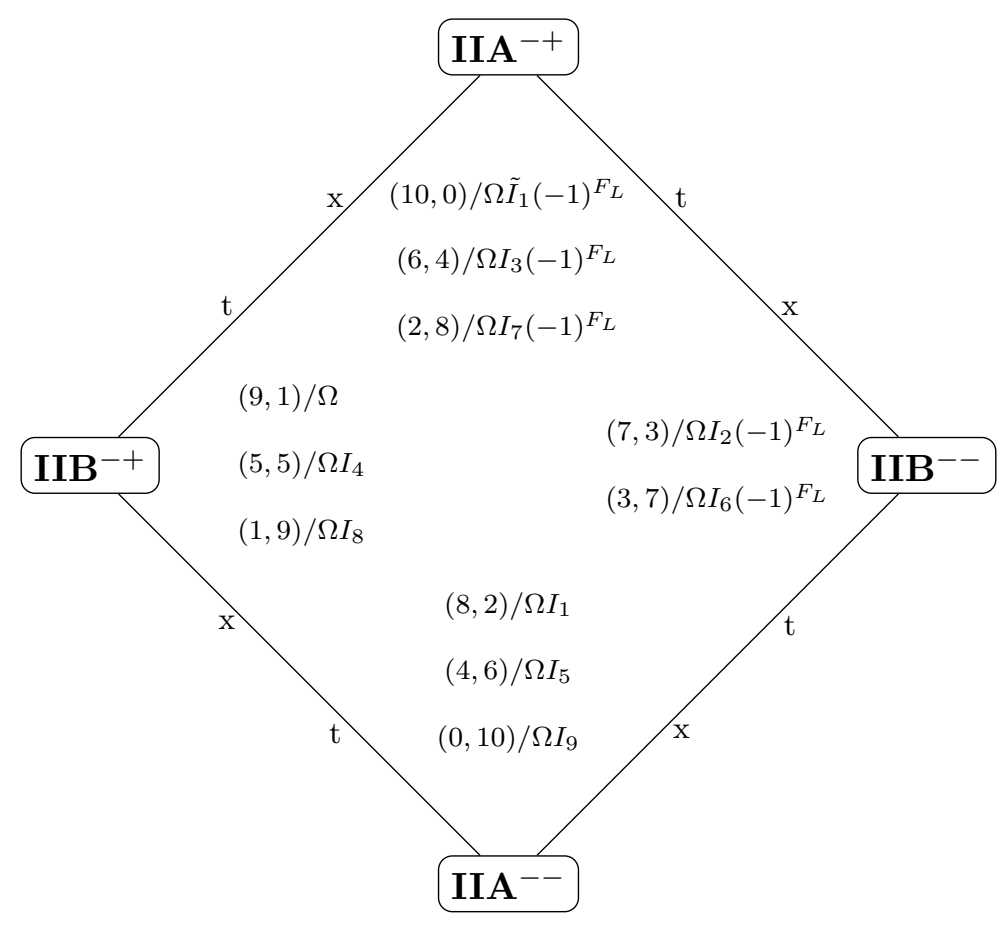

Figure 3. Orientifold projections removing the massless ghosts for Euclidean theories.

\subsection{Speculations and objectives}

In this section, we will make a couple of general and arguably speculative remarks on ghosts. The appearance of ghosts are generally thought to lead to problems for the quantized version of a theory as it spoils the probabilistic interpretation of the wave-function. In the so far discussed closed string sector the massless modes are the graviton and other form fields. The quantum nature of these states has not really been tested experimentally so that here we take the pragmatic point of view of being agnostic whether perturbative ghosts are indeed a disaster for the theory or only indicate whether we should go beyond our usual understanding of a quantum (gravity) theory.

Exotic string theories and Krein spaces? As we have seen, the exotic string theories give rise to a space of states that has indefinite norm. Such generalizations of Hilbert spaces have been considered in the mathematical physics literature and are called Krein spaces. They go back to the early days of quantum mechanics [46, 47] and [48, 49]. Here we are not intending to provide a full review of this field but just want to touch upon a few issues.

A Krein space $\mathcal{K}$ is a complex vector space with an indefinite metric $\langle\cdot \mid \cdot\rangle$ so that $\mathcal{K}$ splits as $\mathcal{K}=\mathcal{K}_{+} \oplus \mathcal{K}_{-}$, where $\mathcal{K}_{+}$and $\mathcal{K}_{-}$are Hilbert spaces. On such a space $\mathcal{K}$ one can introduce an "orthonormal" basis satisfying $\left\langle\psi_{i} \mid \psi_{j}\right\rangle=\eta_{i j}$. In our case, the Hilbert space $\mathcal{K}_{+}$is generated by the non-ghosts and $\mathcal{K}_{-}$by the additional ghosts.

The adjoint of an operator is defined as usual by the condition $\langle\psi \mid A \phi\rangle=\left\langle A^{\sharp} \psi \mid \phi\right\rangle$ which gives $A^{\sharp}=\eta^{-1} A^{\dagger} \eta$. A (pseudo) self-adjoint operator is defined via $A^{\sharp}=A$ and gives rise to 
real expectation values $\langle\psi \mid A \psi\rangle \in \mathbb{R}$. However, the eigenvalues of a self-adjoint operator are generically not real. An operator preserving the product, i.e. $\langle\psi \mid \phi\rangle=\langle U \psi \mid U \phi\rangle$, is called (pseudo) unitary. It satisfies $U^{\sharp} U=1$ which means $U^{\dagger}=\eta U^{-1} \eta^{-1}$. On a Krein space the unit operator can be expressed as $1=\left|\psi_{i}\right\rangle \eta^{i j}\left\langle\psi_{j}\right|$. We expect that the (exotic) string S-matrix is such a (pseudo) unitary operator.

Whether eventually such a quantum theory on a Krein space is a viable possibility for a theory of quantum gravity remains to be seen and goes beyond the scope of this paper. At least we can state that the quantum theory that originates by allowing extra time-like directions in string theory has an already studied mathematical structure.

Objectives. Although the perturbative approach to the exotic string theories is full of pathologies that are not yet completely understood, it has been argued that these are just artifacts of the perturbative approach and will get resolved in the full theories (as they are all dual to the original type IIA/B superstrings). On the other hand it could also well be that these pathologies are a result of having compact time directions (at least at intermediate stages) and that these are simply not allowed in any reasonable physical theory. In the latter case, there would be no point in following these ideas further.

However, we also do not want to miss a potentially interesting new aspect of string theory, as often applying dualities led to new insights into the theory. Moreover, it is at least appealing that despite conceptual pathologies, the formalism per se seems to go through. Thus, in the following we still take a positive attitude and further expand the formalism of exotic string theories to also include the open string sector. The question we are posing is whether a pathological closed string sector with ghosts that admits dS solutions, can nevertheless host a viable effective D-brane theory that by itself obeys the usual requirements for a consistent quantum field theory, i.e. is ghost free and unitary. This subsector could then be considered to be the Standard Model, whose quantum aspects we have direct experimental access to.

\section{CFT description of Euclidean exotic strings}

In this rather technical section we will take a closer look on the Euclidean world-sheet CFTs. After a short review of the closed string construction of [39] we construct the purely Euclidean open string theories including the fermionic sector. We use this to identify the allowed D-branes of the different theories as well as the tension of the branes. In section 5 these results will be confirmed using the map (2.19) inspired by horizon crossing in the presence of negative branes.

\subsection{Basics of CFTs with Euclidean world-sheets}

Let us first present a couple of basic results for the structure of CFTs on purely Euclidean world-sheets. We will see that in this case some extra factors of $i$ appear.

Lorentzian vs. Euclidean world-sheets. When considering purely Euclidean bosonic closed strings we have to thoroughly disentangle the differences between a Lorentzian, a Wick-rotated Euclidean and a purely Euclidean field theory. We could take several 
approaches to quantize the theory, but the differences in the mode algebra of the field become most apparent when using the path integral formalism. Consider the path integral

$$
Z=\int[D \gamma][D X] e^{K S_{\varepsilon}}
$$

where we introduced $K=\{i,-1\}$ with $K=-1$ only in the Wick-rotated case, and $\varepsilon=\{1,-1\}$ labels the Euclidean or Lorentzian world-sheet action. Thus $K=i, \varepsilon=-1$ is the Lorentzian string, $K=-1, \varepsilon=1$ is the Wick-rotated Euclidean string and finally $K=i, \varepsilon=1$ is the purely Euclidean string. Before applying path integral methods we have to bring the action into a quadratic form

$$
S=-\frac{\varepsilon}{2 \pi \alpha^{\prime}} \int \mathrm{d}^{2} \sigma \sqrt{\varepsilon \operatorname{det} \gamma} \eta_{\mu \nu} X^{\mu} \partial^{a} \partial_{a} X^{\nu}
$$

The kinetic operator depends on the world-sheet metric and thus differs for Lorentzian and Euclidean field theories. The two point function has to satisfy the identity

$$
\frac{2 \pi \alpha^{\prime}}{K \varepsilon} \delta_{\mu_{1}}^{\mu_{2}} \delta^{2}\left(\sigma-\sigma^{\prime}\right)=\sqrt{\varepsilon \operatorname{det} \gamma} \eta_{\mu_{1} \nu} \partial^{a} \partial_{a}\left\langle X^{\mu_{2}}(\sigma) X^{\nu}\left(\sigma^{\prime}\right)\right\rangle
$$

where $\sigma, \sigma^{\prime}$ are coordinates on the world-sheet. The kinetic operator on the r.h.s. is in the $\sigma=\left(\sigma_{1}, \sigma_{2}\right)$ coordinates. Let us concentrate for a moment on the two Euclidean field theories. Introducing the usual cylinder world-sheet coordinate

$$
z=e^{\sigma_{1}+i \sigma_{2}}
$$

the equation for the two point function becomes

$$
\frac{2 \pi \alpha^{\prime}}{K} \delta_{\mu_{1}}^{\mu_{2}} \delta^{2}(z-w)=\eta_{\mu_{1} \nu} \partial_{z} \partial_{\bar{z}}\left\langle X^{\mu_{2}}(z, \bar{z}) X^{\nu}(w, \bar{w})\right\rangle .
$$

The solution to this is given by

$$
\left\langle X^{\mu}(z, \bar{z}) X^{\nu}(w, \bar{w})\right\rangle=\eta^{\mu \nu} \frac{\alpha^{\prime}}{K} \log |z-w|^{2} .
$$

We want to derive the mode algebra for the CFT. The action in the above coordinates reads

$$
S=\frac{1}{2 \pi \alpha^{\prime}} \int \mathrm{d}^{2} z \partial X(z, \bar{z}) \cdot \bar{\partial} X(z, \bar{z}),
$$

where the target-space metric is hidden in the multiplication dot. Going through the usual steps for the above action we find holomorphic and anti-holomorphic currents $\partial X^{\mu}(z)$, $\bar{\partial} X^{\mu}(\bar{z})$. Using (4.5) we find for their two-point functions

$$
\left\langle\partial X^{\mu}(z) \partial X^{\nu}(w)\right\rangle=\eta^{\mu \nu} \frac{\alpha^{\prime}}{K} \frac{1}{(z-w)^{2}} .
$$

Expanding the current fields $\partial X^{\mu}(z)=-\sqrt{\frac{\alpha^{\prime}}{2 \varepsilon}} \sum \hat{\alpha}_{m}^{\mu} z^{-m-1}$, the mode algebra reads

$$
\left[\hat{\alpha}_{m}^{\mu}, \hat{\alpha}_{n}^{\nu}\right]=\varepsilon \eta^{\mu \nu} \frac{\alpha^{\prime}}{K} m \delta_{m+n, 0} .
$$

Thus we see that for the Wick-rotated Euclidean string $(K=-1)$ the procedure gives the usual commutation relations, whereas in the purely Euclidean field theory $(K=i)$ the commutation relations get an extra factor of $-i$. 
Closed and open Euclidean strings. From now on we will only be concerned with the purely Euclidean theories, i.e. the world-sheet theories of the $\operatorname{IIA}^{E}$ and $\operatorname{IIB}^{E}$ exotic string theories. To construct the world-sheet theory we follow [39] closely. The action for a free boson is given by

$$
S_{b}=\frac{1}{2 \pi \alpha^{\prime}} \int \mathrm{d}^{2} \sigma \sqrt{\operatorname{det} g} g^{a b} \eta_{\mu \nu} \partial_{a} X^{\mu} \partial_{b} X^{\nu}
$$

The world-sheet metric $g$ is gauge fixed to the flat metric $g_{\sigma_{1} \sigma_{1}}=g_{\sigma_{2} \sigma_{2}}=1$ and light cone coordinates are chosen as

$$
\sigma_{ \pm}=\sigma_{1} \pm i \sigma_{2}
$$

such that the derivatives become

$$
\partial_{ \pm}=\frac{1}{2}\left(\partial_{\sigma_{1}} \mp i \partial_{\sigma_{2}}\right)
$$

We now choose a convenient mode expansion, simplifying the mode algebra as much as possible. In this framework the oscillators will behave as in the usual string theories. The zero modes will be solely responsible for the changes in the physics. The mode expansion of the closed string sector is given by

$$
\begin{aligned}
X^{\mu}\left(\sigma_{1}, \sigma_{2}\right) & =x^{\mu}+\alpha^{\prime} p^{\mu} \sigma_{1}+\sqrt{\frac{\alpha^{\prime}}{2 i}} \sum_{n \neq 0}\left(\frac{\alpha_{n}^{\mu}}{n} e^{-n \sigma^{+}}+\frac{\bar{\alpha}_{n}^{\mu}}{n} e^{-n \sigma^{-}}\right) \\
& =x^{\mu}+\frac{\alpha^{\prime}}{2} p^{\mu} \log \left(|z|^{2}\right)+\sqrt{\frac{\alpha^{\prime}}{2 i}} \sum_{n \neq 0}\left(\frac{\alpha_{n}^{\mu}}{n} z^{-n}+\frac{\bar{\alpha}_{n}^{\mu}}{n} \bar{z}^{-n}\right),
\end{aligned}
$$

so that the mode algebra becomes

$$
\left[x^{\mu}, p^{\nu}\right]=i \eta^{\mu \nu}, \quad\left[\alpha_{m}^{\mu}, \alpha_{n}^{\nu}\right]=\left[\bar{\alpha}_{m}^{\mu}, \bar{\alpha}_{n}^{\nu}\right]=m \delta_{m,-n} \eta^{\mu \nu}
$$

for $m, n \neq 0$. Moreover, one has as usual $\left[\alpha_{m}^{\mu}, \bar{\alpha}_{n}^{\nu}\right]=0$, and the oscillators $\alpha_{m}^{\mu}, \bar{\alpha}_{m}^{\mu}$ commute with the zero modes $x^{\mu}$ and $p^{\mu}$.

Let us make a couple of remarks. To arrive at this standard mode algebra, we have effectively rescaled the standard oscillator modes $\hat{\alpha}$ by a factor of $\sqrt{i}$. As a consequence, one needs to be very careful when computing overlaps of states $\left\langle\phi^{1} \mid \phi^{2}\right\rangle$. Indeed, taking the general definition of the conjugate $\left(\phi_{n}\right)^{\dagger}=\left(\phi^{\dagger}\right)_{-n}$ for a field $\phi$ in Euclidean CFT into account, the rescaling leads to phase factors, as some of the fields won't be purely real anymore. On the one hand, in this paper we are mostly concerned with partition functions where these phases do not matter as one simply counts the number of states at each level. On the other hand, in the boundary state overlaps (to be introduced later in (4.32)), due to loop-channel tree-channel equivalence the (suitably generalized) CPT operator $\Theta$ has to remove these factors. These two facts make this basis very useful for our computations.

If one wants to calculate the low energy effective action and determine for instance the sign of the kinetic terms, one also needs to know the normalization of the corresponding vertex operators. In fact in [39] the normalizations for the metric and the B-field vertex operators have been determined. The graviton state turned out to be

$$
\left|V_{G}(p)\right\rangle=i \epsilon_{\mu \nu} \hat{\alpha}_{-1}^{\mu} \hat{\bar{\alpha}}_{-1}^{\nu}|p\rangle=\epsilon_{\mu \nu} \alpha_{-1}^{\mu} \bar{\alpha}_{-1}^{\nu}|p\rangle
$$


whereas the Kalb-Ramond state has a different normalization

$$
\left|V_{B}(p)\right\rangle=-b_{\mu \nu} \hat{\alpha}_{-1}^{\mu} \hat{\bar{\alpha}}_{-1}^{\nu}|p\rangle=-i b_{\mu \nu} \alpha_{-1}^{\mu} \bar{\alpha}_{-1}^{\nu}|p\rangle .
$$

Thus, working with the modes $\alpha^{\mu}, \bar{\alpha}^{\mu}$ and treating them in the same way as the usual oscillators in string theory makes it evident that the $B$-field is a ghost.

In a similar fashion one can expand the open string into modes. For NeumannNeumann (NN) and Dirichlet-Dirichlet (DD) boundary conditions the mode expansion reads

$$
\begin{aligned}
& X_{\mathrm{NN}}^{\mu}=x^{\mu}+2 \alpha^{\prime} p^{\mu} \sigma_{1}+\sqrt{-2 i \alpha^{\prime}} \sum_{n \neq 0} \frac{\alpha_{n}^{\mu}}{2 n}\left(e^{-n \sigma_{-}}+e^{-n \sigma_{+}}\right), \\
& X_{\mathrm{DD}}^{\mu}=x^{\mu}+\frac{\Delta x^{\mu}}{\pi} \sigma_{2}+\sqrt{-2 i \alpha^{\prime}} \sum_{n \neq 0} \frac{\alpha_{n}^{\mu}}{2 n}\left(e^{-n \sigma_{-}}-e^{-n \sigma_{+}}\right),
\end{aligned}
$$

with the distance between the branes $\Delta x^{\mu}=x_{a}^{\mu}-x_{b}^{\mu}$. Taking the derivatives one gets

$$
\begin{aligned}
& \partial_{ \pm} X_{\mathrm{NN}}^{\mu}=\alpha^{\prime} p^{\mu}-\sqrt{\frac{\alpha^{\prime}}{2 i}} \sum_{n \neq 0} \alpha_{n}^{\mu} e^{-n \sigma_{ \pm}}=-\sqrt{\frac{\alpha^{\prime}}{2 i}} \sum_{n \in \mathbb{Z}} \alpha_{n}^{\mu} e^{-n \sigma_{ \pm}}, \\
& \partial_{ \pm} X_{\mathrm{DD}}^{\mu}=\mp i \frac{\Delta x^{\mu}}{2 \pi} \pm \sqrt{\frac{\alpha^{\prime}}{2 i}} \sum_{n \neq 0} \alpha_{n}^{\mu} e^{-n \sigma_{ \pm}}= \pm \sqrt{\frac{\alpha^{\prime}}{2 i}} \sum_{n \in \mathbb{Z}} \alpha_{n}^{\mu} e^{-n \sigma_{ \pm}},
\end{aligned}
$$

where we have defined the zero modes as

$$
\alpha_{0}^{\mu, \mathrm{NN}}=-p^{\mu} \sqrt{2 i \alpha^{\prime}}, \quad \alpha_{0}^{\mu, \mathrm{DD}}=-\frac{\sqrt{-i}}{\sqrt{2 \alpha^{\prime}} \pi} \Delta x^{\mu} .
$$

For completeness we also present the mode expansion for mixed boundary conditions

$$
\begin{aligned}
& X_{\mathrm{ND}}^{\mu}=x^{\mu}+\sqrt{-2 i \alpha^{\prime}} \sum_{n \in \mathbb{Z}+1 / 2} \frac{\alpha_{n}^{\mu}}{2 n}\left(e^{-n \sigma_{-}}+e^{-n \sigma_{+}}\right), \\
& X_{\mathrm{DN}}^{\mu}=x^{\mu}+\sqrt{-2 i \alpha^{\prime}} \sum_{n \in \mathbb{Z}+1 / 2} \frac{\alpha_{n}^{\mu}}{2 n}\left(e^{-n \sigma_{-}}-e^{-n \sigma_{+}}\right) .
\end{aligned}
$$

Next we want to define the closed and open string partition functions. For that purpose we first focus just on a single direction $X\left(\sigma_{1}, \sigma_{2}\right)$ and recall that in the Sugawara construction the energy momentum tensor reads

$$
T(z)=\frac{i}{\alpha^{\prime}} \eta_{\mu \nu}: \partial X^{\mu}(z) \partial X^{\nu}(z): .
$$

With this, the normal ordered Hamiltonian becomes

$$
H=\int_{0}^{2 \pi} \frac{\mathrm{d} \sigma}{2 \pi \alpha^{\prime}}\left(\left(\partial_{+} X\right)^{2}+\left(\partial_{-} X\right)^{2}\right)=-i\left(L_{0}+\bar{L}_{0}-\frac{c}{12}\right)
$$


where the factor of $i$ originates in the mode expansion. The explicit form of the energy momentum tensor's zero mode $L_{0}$ is

$$
L_{0}=i \frac{\alpha^{\prime} p^{2}}{4}+\sum_{n>0} \eta_{\mu \nu} \alpha_{-n}^{\mu} \alpha_{n}^{\nu}
$$

and similarly for $\bar{L}_{0}$. The second term is just the number operator which has non-negative integer eigenvalues. In contrast to the usual case, the zero mode contribution is purely imaginary.

The momentum $P$ which generates $\sigma_{2}$ translations is now given by

$$
P=-i \int_{0}^{2 \pi} \frac{\mathrm{d} \sigma}{2 \pi \alpha^{\prime}}\left(\left(\partial_{+} X\right)^{2}-\left(\partial_{-} X\right)^{2}\right)=-\left(L_{0}-\bar{L}_{0}\right) .
$$

In this case the normal ordering constant cancels out. As a consequence the torus and cylinder amplitudes receive additional factors of $i$. The torus amplitude with complex structure $\tau=\tau_{1}+i \tau_{2}$ is constructed by taking a field theory on a circle, translating in $\sigma_{1}$ direction by $\tau_{2}$, in $\sigma_{2}$ direction by $\tau_{1}$ and identifying the ends, producing the trace. With

$$
q=e^{2 \pi i\left(\tau_{1}+i \tau_{2}\right)}
$$

the torus partition function can be written as

$$
Z^{\text {torus }}=\operatorname{Tr}\left(e^{-2 \pi i \tau_{2} H-2 \pi i \tau_{1} P}\right)=\operatorname{Tr}\left(q^{L_{0}-\frac{c}{24}} \bar{q}^{\bar{L}_{0}-\frac{c}{24}}\right) .
$$

Note that due to the missing Wick rotation for the Euclidean CFT, the coefficient in front of the Hamiltonian is $-2 \pi i$ instead of the usual $-2 \pi$. But this factor gets multiplied by the additional factor of $-i$ in the Hamiltonian (4.22), such that the expression for the partition function is still the usual one. Evaluating the amplitude for a single direction one obtains

$$
Z^{\text {torus }}=\frac{e^{i \pi / 4}}{\sqrt{4 \pi \alpha^{\prime} \tau_{2}}|\eta(\tau)|^{2}},
$$

reproducing the result of [39].

Now we turn to the open string cylinder amplitude which is defined as

$$
Z^{\mathcal{C}}(t)=\operatorname{Tr}\left(e^{-2 \pi i t H_{\mathrm{open}}}\right)=\operatorname{Tr}\left(e^{-2 \pi t\left(L_{0}-\frac{c}{24}\right)}\right),
$$

with $t$ the circumference of the cylinder. The explicit form of $L_{0}$ for NN and DD boundary conditions is

$$
L_{0}^{\mathrm{NN}}=i \alpha^{\prime} p^{2}+\sum_{n>0} \eta_{\mu \nu} \alpha_{-n}^{\mu} \alpha_{n}^{\nu}, \quad L_{0}^{\mathrm{DD}}=\frac{i}{4 \pi^{2} \alpha^{\prime}} Y^{2}+\sum_{n>0} \eta_{\mu \nu} \alpha_{-n}^{\mu} \alpha_{n}^{\nu} .
$$

The total distance between the Dirichlet loci is defined as $Y^{2}=\eta_{\mu \nu} \Delta x^{\mu} \Delta x^{\nu}$. Now, considering only a single direction of either NN or DD type, the open string partition functions can be evaluated straightforwardly with the result

$$
Z^{\mathcal{C}(\mathrm{NN})}(t)=\frac{e^{-i \pi / 4}}{\sqrt{2 \alpha^{\prime} t} \eta(i t)}, \quad Z^{\mathcal{C}(\mathrm{DD})}(t)=e^{-\frac{i t}{2 \pi \alpha^{\prime}} Y^{2}} \frac{1}{\eta(i t)} .
$$


The additional factor of $e^{-i \pi / 4}$ in the Neumann-Neumann case arises from the analytic continuation of the Gaussian integral for the zero mode. ${ }^{8}$ For mixed boundary conditions one finds

$$
Z^{\mathcal{C}(\mathrm{ND})}(t)=\sqrt{\frac{2 \eta(i t)}{\theta_{4}(i t)}} .
$$

As usual, the open string (loop-channel) cylinder amplitude is closely related to the (treechannel) overlap of boundary states

$$
\tilde{Z}(l)=\left\langle\Theta B\left|e^{2 \pi i l H_{\text {closed }}}\right| B\right\rangle=\left\langle\Theta B\left|e^{-2 \pi l\left(L_{0}+\bar{L}_{0}-\frac{c}{12}\right)}\right| B\right\rangle,
$$

with $l$ the length of the cylinder formed by the closed strings exchanged between the boundaries. We will construct the appropriate boundary states in the next section.

\subsection{Boundary states}

Next we analyze the construction of boundary states in a Euclidean world-sheet CFT. ${ }^{9}$ For the moment we assume also a purely Euclidean space-time and postpone the treatment of the effects of the target-space metric signature to the next section. The boundary conditions are unaffected by the signature of the world-sheet. Despite the now Euclidean signature we will think of the coordinate $\sigma_{1} \in(0, l)$ as the time coordinate and $\sigma_{2} \in(0, \pi)$ as the space component. The conformal map exchanging the open and closed channels acting on the complexified coordinate $\xi=\sigma_{2}+i \sigma_{1}$ is then given by

$$
f(\xi)=-i \frac{\pi}{l} \xi
$$

which is the same as in the Lorentzian case exchanging world-sheet time $\tau$ and space $\sigma$. The Neumann and Dirichlet gluing conditions are given by

$$
\left.\partial_{\sigma_{1}} X^{\mu}\right|_{\sigma_{1}=0}\left|B_{N}\right\rangle=0,\left.\quad \partial_{\sigma_{2}} X^{\mu}\right|_{\sigma_{1}=0}\left|B_{D}\right\rangle=0 .
$$

Inserting the mode expansion (4.13) results in

$$
p^{\mu}\left|B_{N}\right\rangle=0, \quad\left(\alpha_{n}^{\mu}+\bar{\alpha}_{-n}^{\mu}\right)\left|B_{N}\right\rangle=0
$$

as well as

$$
x^{\mu}\left|B_{D}\right\rangle=y^{\mu}, \quad\left(\alpha_{n}^{\mu}-\bar{\alpha}_{-n}^{\mu}\right)\left|B_{D}\right\rangle=0,
$$

where $y^{\mu}$ is the position of the brane. These are exactly the same conditions as in the Lorentzian case. Defining a matrix $S_{\mu \nu}= \pm \eta_{\mu \nu}$, with the + sign for Neumann directions and the - sign for Dirichlet directions, the non-zero mode conditions are given by

$$
\left(\alpha_{n}^{\mu}+S_{\nu}^{\mu} \bar{\alpha}_{-n}^{\nu}\right)|B\rangle=0 .
$$

\footnotetext{
${ }^{8}$ We often employed the Gaussian integral $\int_{-\infty}^{\infty} \mathrm{d} x e^{-a x^{2}+b x}=\sqrt{\frac{\pi}{a}} \cdot e^{\frac{b^{2}}{4 a}}$ and its analytic continuation. This is where most of the phases arise.

${ }^{9}$ Our analysis follows that of [50-53] for Lorentzian strings.
} 
As usual the solution to these gluing conditions is

$$
|B\rangle=\frac{1}{\mathcal{N}} \exp \left(-\sum_{n=1}^{\infty} \frac{1}{n} \alpha_{-n}^{\mu} S_{\mu \nu} \bar{\alpha}_{-n}^{\nu}\right)|0\rangle
$$

Using the explicit form of the boundary states (4.38), the overlap (4.32) becomes

$$
\tilde{Z}^{\mathcal{C}(\mathrm{NN})}(l)=\frac{1}{\mathcal{N}_{N}^{2}} \frac{1}{\eta(2 i l)} .
$$

Mapping the open string loop-channel result with $t=1 / 2 l$ and a modular S transformation to the closed string tree-channel, the normalization constant can be determined via

$$
Z^{\mathcal{C}(\mathrm{NN})}(t)=\frac{e^{-i \pi / 4}}{\sqrt{2 \alpha^{\prime} t} \eta(i t)}=\frac{e^{-i \pi / 4}}{\sqrt{\alpha^{\prime}} \eta(2 i l)} \stackrel{!}{=} \frac{1}{\mathcal{N}_{N}^{2} \eta(2 i l)} \Rightarrow \mathcal{N}_{N}=\left(\alpha^{\prime}\right)^{1 / 4} e^{i \pi / 8} .
$$

Turning to the DD case, the only thing that changes is the $\alpha_{0}$ zero-mode contribution which is now given by

$$
\begin{aligned}
& \int_{-\infty}^{\infty} \frac{d k_{a} d k_{b}}{2 \pi} e^{i k_{a} x_{a}} e^{i k_{b} x_{b}}\left\langle k_{a}\left|e^{-2 \pi l\left(\alpha_{0}\right)^{2}}\right| k_{b}\right\rangle \\
& =\int_{-\infty}^{\infty} d k_{a} d k_{b} e^{i k_{a} x_{a}} e^{i k_{b} x_{b}} e^{-\pi i l \alpha^{\prime} k_{b}^{2}} \delta\left(k_{a}+k_{b}\right) \\
& =\int_{-\infty}^{\infty} d k_{a} e^{i k_{a}\left(x_{a}-x_{b}\right)} e^{-\pi i l \alpha^{\prime} k_{a}^{2}}=\frac{e^{i \pi / 4}}{\sqrt{\alpha^{\prime} l}} e^{-i \frac{Y^{2}}{4 \pi \alpha^{\prime} l}},
\end{aligned}
$$

where we used $\langle 0 \mid 0\rangle=2 \pi \delta(0)$ and that the CPT operator $\Theta$ in (4.32) involves a complex conjugation. Therefore the total DD overlap is

$$
\tilde{Z}^{\mathcal{C}(\mathrm{DD})}(l)=\frac{e^{-i \pi / 4}}{\mathcal{N}_{D}^{2}} \frac{1}{\sqrt{4 \pi^{2} \alpha^{\prime} l} \eta(2 i l)} e^{-i \frac{Y^{2}}{4 \pi \alpha^{\prime} l}} .
$$

Comparing this to the open string amplitude we obtain

$$
\mathcal{N}_{D}=\left(\alpha^{\prime}\right)^{-1 / 4} e^{-i \pi / 8} .
$$

Finally, as a cross-check for the normalization factors, we evaluate the mixed case as

$$
\tilde{Z}^{\mathcal{C}(\mathrm{ND})}(l)=\frac{1}{\mathcal{N}_{N} \mathcal{N}_{D}} \sqrt{\frac{2 \eta(2 i l)}{\theta_{2}(2 i l)}}=\sqrt{\frac{2 \eta(i t)}{\theta_{4}(i t)}}=Z^{\mathcal{C}(\mathrm{ND})}(t),
$$

featuring that the normalizations of the boundary states are indeed consistent.

The total cylinder amplitudes. After having studied the open string amplitude for just a single direction, we now combine the separate contributions into a total cylinder amplitude of two parallel $d$-dimensional branes in $D$ space-time dimensions. ${ }^{10}$ For the open string loop-channel amplitude one obtains

$$
\mathcal{A}=V_{d} \int_{0}^{\infty} \frac{d t}{2 t}\left(\frac{e^{-i \pi / 4}}{\sqrt{8 \pi^{2} \alpha^{\prime} t}}\right)^{d} \frac{1}{\eta^{D-2}(i t)} e^{-\frac{i t}{2 \pi \alpha^{\prime}} Y^{2}}
$$

\footnotetext{
${ }^{10}$ Note that in this convention a Dp-brane has $d=p+1$.
} 
where the additional $\eta^{2}(i t)$ factor originates from the ghost contribution. The total closed string tree-channel amplitude is

$$
\tilde{\mathcal{A}}=\frac{V_{d}}{\mathcal{N}^{2}} \int_{0}^{\infty} d l e^{i \pi(D-d) / 4}\left(\frac{1}{4 \pi^{2} \alpha^{\prime} l}\right)^{(D-d) / 2} \frac{1}{\eta^{D-2}(2 i l)} e^{-\frac{i}{4 \pi \alpha^{\prime} l} Y^{2}} .
$$

Applying a modular S-transformation, this amplitude is mapped to the loop-channel and comparing it to (4.45) one can read off the normalization

$$
\mathcal{N}^{-1}=2^{\frac{D-2}{4}} e^{\frac{i \pi}{8}(D-2 d)}\left(4 \pi^{2} \alpha^{\prime}\right)^{\frac{1}{4}(D-2 d-2)} .
$$

Finally, the tension of the branes is determined by the coupling of the boundary state to a graviton with polarization $\epsilon_{\mu \nu}$

$$
\left\langle V_{g} \mid B\right\rangle=-\frac{1}{\mathcal{N}}\left\langle 0\left|\epsilon_{\mu \nu} S^{\mu \nu}\right| 0\right\rangle=-\frac{1}{\mathcal{N}} \epsilon_{\mu \nu} S^{\mu \nu} V_{d+1} \stackrel{!}{=}-T_{d} \epsilon_{\mu \nu} S^{\mu \nu} V_{d+1},
$$

so that the tension is given by the normalization of the boundary state as $T_{d}=\mathcal{N}^{-1}$. For connecting a D-brane theory to phenomenology, we require the tension to be real, so that the normalization of the boundary state also has to be real. Inserting $D=10$ into (4.47), we see that there are exactly three cases fulfilling this condition, $d \in\{1,5,9\}$ with tension

$$
T_{d}= \pm 2^{2}\left(4 \pi^{2} \alpha^{\prime}\right)^{(4-d) / 2},
$$

with the minus sign for $d \in\{1,9\}$ and the plus sign for $d=5$.

\subsection{Fermionic boundary states}

So far we have only discussed the contribution of the world-sheet bosons to the boundary states. Let us now also discuss the inclusion of the world-sheet fermions. The action for a free fermion is

$$
S_{f}=\frac{i \epsilon}{4 \pi} \int \mathrm{d}^{2} \sigma \sqrt{\epsilon \operatorname{det} g} \eta_{\mu \nu} \bar{\Psi}^{\mu} \gamma^{\alpha} \partial_{\alpha} \Psi^{\nu},
$$

where the $2 \times 2$ matrices $\gamma^{\alpha}$ satisfy the Clifford algebra with respect to the world-sheet metric $g_{\alpha \beta}$

$$
\left\{\gamma^{\alpha}, \gamma^{\beta}\right\}=2 g^{\alpha \beta} \mathbb{1}_{2}
$$

Moreover, one defines

$$
\begin{array}{ll}
\bar{\Psi}^{\mu}=\Psi^{\mu} \gamma^{0} & \text { in the Lorentzian case and } \\
\bar{\Psi}^{\mu}=\Psi^{\mu} C & \text { in the Euclidean case, }
\end{array}
$$

with $C$ the charge conjugation matrix

$$
\left(\gamma^{\alpha}\right)^{\top}=C \gamma^{\alpha} C^{-1}, \quad C^{\top}=C^{\dagger}=C^{-1}=C .
$$

For Lorentzian signature we choose the representation

$$
\gamma^{0}=\left(\begin{array}{ll}
0 & 1 \\
1 & 0
\end{array}\right)=\hat{\sigma}_{1}, \quad \gamma^{1}=\left(\begin{array}{cc}
0 & 1 \\
-1 & 0
\end{array}\right) .
$$


Under Wick rotation $\tau=i \sigma_{1}$ one has $\partial_{\tau} \rightarrow-i \partial_{\sigma_{1}}$, so effectively $\gamma^{1}$ is replaced with

$$
\gamma^{1}=\left(\begin{array}{cc}
0 & -i \\
i & 0
\end{array}\right)=\hat{\sigma}_{2} .
$$

Therefore, the Wick rotation has the effect of replacing the Lorentzian gamma matrices with the Euclidean gamma matrices. Then, the kinetic term of the Wick rotated theory is the same as in the purely Euclidean theory up to an overall sign. Choosing the same Pauli matrices $\hat{\sigma}_{1}, \hat{\sigma}_{2}$ also as a representation for the Euclidean Clifford algebra, the conditions (4.53) uniquely determine $C$ to be

$$
C=\left(\begin{array}{cc}
0 & -1 \\
-1 & 0
\end{array}\right)=-\gamma^{0} .
$$

Denoting the components of a $2 \mathrm{D}$ spinor as $\Psi^{\mu}=\left(\Psi_{+}^{\mu}, \Psi_{-}^{\mu}\right)^{\top}$, the action reduces to

$$
S_{f}=-\frac{K}{2 \pi} \int \mathrm{d}^{2} \sigma \eta_{\mu \nu}\left(\Psi_{+}^{\mu} \partial_{-} \Psi_{+}^{\nu}+\Psi_{-}^{\mu} \partial_{+} \Psi_{-}^{\nu}\right)
$$

where $K=-1$ for the (Wick-rotated) Lorentzian world-sheet and $K=i$ for the Euclidean case. Here we have used again the coordinates $\sigma^{ \pm}=\sigma_{1} \pm i \sigma_{2}$. The equations of motion are

$$
\partial_{-} \Psi_{+}^{\mu}=\partial_{+} \Psi_{-}^{\mu}=0
$$

with the usual (anti-)holomorphic solutions $\Psi_{+}^{\mu}=\Psi_{+}^{\mu}\left(\sigma^{+}\right)$and $\Psi_{-}^{\mu}=\Psi_{-}^{\mu}\left(\sigma^{-}\right)$, which can be expanded as

$$
\Psi_{+}^{\mu}=\sqrt{-K} \sum_{r} b_{r}^{\mu} e^{-2 \pi i r \sigma^{+}}, \quad \Psi_{-}^{\mu}=\sqrt{-K} \sum_{r} \bar{b}_{r}^{\mu} e^{-2 \pi i r \sigma^{-}} .
$$

As in the bosonic case, the factor $\sqrt{-K}$ ensures that the mode algebra takes the usual form

$$
\left\{b_{r}^{\mu}, b_{s}^{\nu}\right\}=\delta_{r,-s} \eta^{\mu \nu}, \quad\left\{\bar{b}_{r}^{\mu}, \bar{b}_{s}^{\nu}\right\}=\delta_{r,-s} \eta^{\mu \nu} \quad\left\{b_{r}^{\mu}, \bar{b}_{s}^{\nu}\right\}=0
$$

The energy momentum tensor is obtained by the Sugawara construction, resulting in the explicit expression for the zero mode

$$
L_{0}=\sum_{r \geq 1 / 2}\left(r+\frac{1}{2}\right) \eta_{\mu \nu} b_{-r}^{\mu} b_{r}^{\nu}
$$

Now that we have the algebra of the fermions we turn to the construction of the boundary state. We will work again in the Euclidean formalism. The exchange of $\sigma_{1}$ and $\sigma_{2}$ acts on the Euclidean light cone variables as

$$
\sigma^{ \pm} \rightarrow \sigma^{\prime \pm}=\mp i \sigma^{ \pm}
$$

The fermions transform under this conformal transformation as

$$
\Psi_{ \pm}^{\prime}\left(\sigma^{\prime \pm}\right)=\left(\frac{\partial \sigma^{\prime \pm}}{\partial \sigma^{ \pm}}\right)^{-1 / 2} \Psi_{ \pm}\left(\sigma^{ \pm}\right)
$$


Imposing the open string boundary conditions on the boundary state, and taking the transformation behavior into account one obtains conditions on the boundary states

$$
\left(\Psi_{+}^{\mu}\left(\sigma^{+}\right)+i \eta S_{\nu}^{\mu} \Psi_{-}^{\nu}\left(\sigma^{-}\right)\right)|B, \eta\rangle=0,
$$

where $\eta= \pm 1$ labels periodic/antiperiodic boundary conditions. Expanding into modes results in the fermionic gluing conditions

$$
\left(b_{r}^{\mu}+i \eta S_{\nu}^{\mu} \bar{b}_{-r}^{\nu}\right)|B, \eta\rangle=0 .
$$

As usual, these gluing conditions are solved by the state

$$
|B, \eta\rangle_{\mathrm{NS}}=\frac{1}{\mathcal{N}} \exp \left(-i \eta \sum_{r=1 / 2}^{\infty} b_{-r}^{\mu} S_{\mu \nu} \bar{b}_{-r}^{\nu}\right)|0\rangle
$$

in the NS sector and by

$$
|B, \eta\rangle_{\mathrm{R}}=\frac{1}{\mathcal{N}} \exp \left(-i \eta \sum_{n=1}^{\infty} b_{-n}^{\mu} S_{\mu \nu} \bar{b}_{-n}^{\nu}\right)|0\rangle_{\mathrm{R}}
$$

in the R sector, where $|0\rangle_{R}$ is the Ramond ground state which satisfies the gluing conditions for the zero modes. The resulting tree-channel annulus amplitudes for a single fermion read

$$
\begin{aligned}
\left\langle B, \eta\left|e^{-2 \pi l\left(L_{0}+\bar{L}_{0}-\frac{c}{12}\right)}\right| B, \eta\right\rangle_{\mathrm{NS}} & =\sqrt{\frac{\theta_{3}(2 i l)}{\eta(2 i l)}}, \\
\left\langle B, \eta\left|e^{-2 \pi l\left(L_{0}+\bar{L}_{0}-\frac{c}{12}\right)}\right| B,-\eta\right\rangle_{\mathrm{NS}} & =\sqrt{\frac{\theta_{4}(2 i l)}{\eta(2 i l)}}, \\
\left\langle B, \eta\left|e^{-2 \pi l\left(L_{0}+\bar{L}_{0}-\frac{c}{12}\right)}\right| B, \eta\right\rangle_{\mathrm{R}} & =\sqrt{\frac{\theta_{2}(2 i l)}{\eta(2 i l)}}, \\
\left\langle B, \eta\left|e^{-2 \pi l\left(L_{0}+\bar{L}_{0}-\frac{c}{12}\right)}\right| B,-\eta\right\rangle_{\mathrm{R}} & =0 .
\end{aligned}
$$

Applying a modular S-transformation leads to the open channel amplitudes

$$
\begin{aligned}
\operatorname{Tr}_{\mathrm{NS}}\left(e^{-2 \pi t\left(L_{0}-\frac{c}{24}\right)}\right) & =\sqrt{\frac{\theta_{3}(i t)}{\eta(i t)}}, \\
\operatorname{Tr}_{\mathrm{NS}}\left((-1)^{F} e^{-2 \pi t\left(L_{0}-\frac{c}{24}\right)}\right) & =\sqrt{\frac{\theta_{4}(i t)}{\eta(i t)}}, \\
\operatorname{Tr}_{\mathrm{R}}\left(e^{-2 \pi t\left(L_{0}-\frac{c}{24}\right)}\right) & =\sqrt{\frac{\theta_{2}(i t)}{\eta(i t)}}, \\
\operatorname{Tr}_{\mathrm{R}}\left((-1)^{F} e^{-2 \pi t\left(L_{0}-\frac{c}{24}\right)}\right) & =0,
\end{aligned}
$$

which are the same as for Lorentzian superstrings. 
Now let us construct the boundary state for a full d-dimensional D-brane in 10D. As usual, invariance of the boundary states under the left and right GSO projections and stability requires the presence of all sectors. Then the total loop-channel annulus amplitude for two parallel $d$-dimensional branes becomes

$$
A=V_{d} \int_{0}^{\infty} \frac{d t}{2 t}\left(\frac{1}{\sqrt{8 \pi^{2} \alpha^{\prime} t}}\right)^{d} e^{i \pi d / 4} \frac{\theta_{3}^{4}(i t)-\theta_{4}^{4}(i t)-\theta_{2}^{4}(i t)}{\eta^{12}(i t)} e^{-\frac{i t}{2 \pi \alpha^{\prime}} Y^{2}} .
$$

Transforming this amplitude to the closed tree-channel amplitude

$$
\tilde{A}=V_{d} \int_{0}^{\infty} d l\left(\frac{\sqrt{l}}{\sqrt{4 \pi^{2} \alpha^{\prime}}}\right)^{d} e^{i \pi d / 4} \frac{\theta_{3}^{4}(2 i l)-\theta_{2}^{4}(2 i l)-\theta_{4}^{4}(2 i l)}{\eta^{12}(2 i l) \cdot(\sqrt{2 l})^{8}} e^{-\frac{i}{4 \pi \alpha^{\prime} l} Y^{2}}
$$

allows us to fix the relative contribution from the boundary states as

$$
|D\rangle=\frac{1}{2 \mathcal{N}}\left(|B,-\rangle_{\mathrm{NS}}-|B,+\rangle_{\mathrm{NS}}+i|B,+\rangle_{\mathrm{R}}+i|B,-\rangle_{\mathrm{R}}\right),
$$

where the normalization $\mathcal{N}$ is the same as in the bosonic case.

\subsection{The influence of space-time signature}

In the CFT approach the signature of the space-time merely appears as a sign change in the commutation relations. This is hidden in most formulas we have written down so far. In this section we will take a closer look how the signature influences the amplitudes and boundary states.

We have seen that after absorbing the factor $K=i$ in a redefinition of the modes, we essentially get back the results for the Lorentzian string. The only difference resides in the zero mode contribution. As we will be concerned with branes wrapping various amounts of time dimension, in the following a $\mathrm{D}_{(10-p, p)}^{(s, t)}$-brane will fill $t$ time and $s$ space dimensions in a $\mathbb{R}^{10-p, p}$ target-space with $p$ time and $10-p$ space dimensions. Thus the system we are concerned with consists of

- $N_{t}=t$ time dimensions with Neumann boundary conditions,

- $D_{t}=p-t$ time dimensions with Dirichlet boundary conditions,

- $N_{s}=s$ space dimensions with Neumann boundary conditions,

- $D_{s}=10-p-s$ space dimensions with Dirichlet boundary conditions.

In the analysis so far all directions were assumed to be space-like. Let us now analyze what changes in case some of the directions become time-like. First, recall that the oscillator part of the boundary state (4.38) involves the matrix $S_{\mu \nu}$. For a $\mathrm{D}_{(10-p, p)}^{(s, t)}$-brane this takes the form

$$
S=\left(\begin{array}{llll}
\mathbb{1}_{N_{s}} & & & \\
& -\mathbb{1}_{N_{t}} & & \\
& & -\mathbb{1}_{D_{s}} & \\
& & & \mathbb{1}_{D_{t}}
\end{array}\right) .
$$


Thus, we see that the oscillators of a space-like N/D direction contribute to the boundary state like a $\mathrm{D} / \mathrm{N}$ time-like direction. However, these signs in $S_{\mu \nu}$ cancel anyway when computing the overlap.

Now, let us consider the zero mode contribution, where some phase factors appeared from the zero mode integrals. For a Neumann boundary condition in a space-like direction this phase is

$$
\mathcal{N}_{N, \text { space }}^{-2} \propto \int_{0}^{\infty} \mathrm{d} p e^{-\pi i p^{2}}=e^{-i \pi / 4}
$$

Changing the signature replaces $p^{2}$ by $-p^{2}$, so that

$$
\mathcal{N}_{N, \text { time }}^{-2} \propto \int_{0}^{\infty} \mathrm{d} p e^{\pi i p^{2}}=e^{i \pi / 4}
$$

Similarly, for a Dirichlet direction the exact same integrals appear in the overlap of the zero modes of the boundary states, i.e. for a space-like direction the phase

$$
\begin{aligned}
\mathcal{N}_{D, \text { space }}^{2} \propto \int_{0}^{\infty} \mathrm{d} p e^{-\pi i p^{2}} & =e^{-i \pi / 4} \\
\Rightarrow & \mathcal{N}_{D, \text { time }}^{2} \propto \int_{0}^{\infty} \mathrm{d} p e^{\pi i p^{2}}=e^{i \pi / 4}
\end{aligned}
$$

appears. This implies that changing the signature, the only effect on the normalization of the boundary state is a change of the phase factor such that

$$
\begin{aligned}
\arg \left(\mathcal{N}_{N, \text { space }}\right) & =\arg \left(\mathcal{N}_{D \text {,time }}\right), \\
\arg \left(\mathcal{N}_{N, \text { time }}\right) & =\arg \left(\mathcal{N}_{D \text {,space }}\right) .
\end{aligned}
$$

Effectively this means that the formula for the normalization (4.47) holds in all signatures, one just has to adjust the phase factor as

$$
T_{(10-p, p)}^{(s, t)}=2^{2} e^{\frac{i \pi}{4}(5+t-p-s)}\left(4 \pi^{2} \alpha^{\prime}\right)^{\frac{1}{2}(4-s-t)} .
$$

Note that we have simply replaced $d \rightarrow \tilde{d}=d+D_{t}-N_{t}=p+s-t$ in the phase factor to account for the additional phases. This formula is now valid for all branes in Euclidean world-sheet theories.

As a check let us consider a (positive) Dp-brane on top of a negative $\widehat{\mathrm{Dp}}$-brane from section 2.3. Crossing the interface of the negative brane, the type II Dp-brane becomes a $\mathrm{D}_{(10-p, p)}^{(1, p)}$-brane in the respective exotic superstring theory. According to (4.78), the tension $T_{(10-p, p)}^{(1, p)}=-T_{\mathrm{Dp}}$ changes sign, which is consistent with the map (2.19). The map also implies that the R-R charge of the brane has changed sign. ${ }^{11}$

Taking now into account that the tension is real only for $\tilde{d} \in\{1,5,9\}$, it is straightforward to iterate all possible (real) branes for a given space-time signature. In the appendix

\footnotetext{
${ }^{11}$ The precise sign of the coupling $\mu_{(10-p, p)}^{(s, t)}$ of the corresponding boundary state to the correctly normalized R-R form is not so easy to determine. Since we do not need it in the following, we refrain from going through the exercise.
} 


\begin{tabular}{|ccccc|}
\hline $\mathrm{s}$ & $\mathrm{t}$ & Tension & $\mathrm{Dp}$ & $\mathrm{E} / \mathrm{L}$ \\
\hline 0 & 2 & - & $\mathrm{D} 1$ & $\mathrm{E}$ \\
1 & 3 & - & D3 & $\mathrm{L}$ \\
6 & 0 & - & $\mathrm{D} 5$ & $\mathrm{E}$ \\
7 & 1 & - & $\mathrm{D} 7$ & $\mathrm{~L}$ \\
2 & 0 & + & $\mathrm{D} 1$ & $\mathrm{E}$ \\
3 & 1 & + & $\mathrm{D} 3$ & $\mathrm{~L}$ \\
4 & 2 & + & D5 & $\mathrm{E}$ \\
5 & 3 & + & D7 & $\mathrm{L}$ \\
\hline
\end{tabular}

\begin{tabular}{|ccccc|}
\hline $\mathrm{s}$ & $\mathrm{t}$ & Tension & Dp & $\mathrm{E} / \mathrm{L}$ \\
\hline 0 & 4 & - & D3 & E \\
1 & 5 & - & D5 & L \\
5 & 1 & - & D5 & L \\
4 & 0 & - & D3 & E \\
0 & 0 & + & D(-1) & E \\
1 & 1 & + & D1 & L \\
2 & 2 & + & D3 & E \\
3 & 3 & + & D5 & L \\
4 & 4 & + & D7 & E \\
5 & 5 & + & D9 & L \\
\hline
\end{tabular}

Table 3. Brane spectrum for signature $(7,3)$ (left) and signature $(5,5)$ (right).

we present an exhaustive list of all D-branes in all possible Euclidean string theories. Here, let us just discuss two examples of space-time signature $(7,3)$ and $(5,5)$.

In the first case there are 3 time directions, thus $p=3$. Then, $\tilde{d} \in\{1,5,9\}$ requires that $s-t$ is either $-2,2$ or 6 . Moreover $s$ and $t$ count the number of longitudinal dimensions of the brane, which cannot exceed the available dimensions, i.e. in this case $0 \leq t \leq 3$, $0 \leq s \leq 7$. Iterating over all possibilities one finds the allowed branes and tensions as shown on the left in table 3 . Note that the tensions are given by (4.78), here we just list the signs. Now we turn to the second example with signature $(5,5)$. As $p=5$, from $\tilde{d} \in\{1,5,9\}$ follows that $s-t$ is either equal to $-4,0$ or 4 . Moreover, $s$ and $t$ are integers in the interval $[0,5]$. Iterating over all possibilities we find the brane spectrum listed on the right in table 3 .

As one can see, only even dimensional branes exist, implying that we are in a type IIB setup. Note that this information was not put in by hand, but is enforced by the signature of space-time. Let us already comment that these tables agree precisely with the results from the next section where a different target-space argument is given for the existence of D-branes in Euclidean exotic superstring theories (see tables 4 and 10). Moreover, the tables are consistent with the classification of D-branes reviewed in tables 1 and 2 .

As a final remark we note that in our derivation the constraints for the allowed D-branes followed directly from the bosonic normalization factor. We have not explicitly discussed the GSO projections in the fermionic sector, but as usual the constraint on even/odd dimension of the branes follows directly from the Clifford algebra of the fermionic zero modes. This computation does not change in the Euclidean case so that the D-branes obtained from the bosonic normalization are also GSO invariant.

\subsection{Orientifolds of Euclidean strings}

In this section, we will take a look at orientifold projections of the Euclidean exotic superstring theories. As the calculation strongly resembles the usual one, we will be very brief and refer to standard textbooks $[52,54]$ for more details. Here we only show that in 
the computation of the loop-channel Klein-bottle and Möbius strip amplitudes, the same phase factors appear as for the corresponding annulus amplitude.

Thus, let us consider a single bosonic direction $X\left(\sigma_{1}, \sigma_{2}\right)$. The orientifold projection $\Omega:\left(\sigma_{1}, \sigma_{2}\right) \rightarrow\left(\sigma_{1},-\sigma_{2}\right)$ acts on the modes as

$$
\Omega \alpha_{n} \Omega^{-1}=\bar{\alpha}_{n} .
$$

One can also combine $\Omega$ with the reflection $I_{1}: X \rightarrow-X$ so that

$$
\left(\Omega I_{1}\right) \alpha_{n}\left(\Omega I_{1}\right)^{-1}=-\bar{\alpha}_{n} .
$$

Moreover, we choose the action of $\Omega$ on the vacuum as $\Omega|0\rangle=|0\rangle$. Recall that the Klein bottle amplitude is defined as

$$
Z_{\Omega}^{\mathcal{K}}=\operatorname{Tr}\left(\Omega q^{L_{0}-c / 24} \bar{q}^{\bar{L}_{0}-c / 24}\right)=\operatorname{Tr}_{\mathrm{sym}}\left(e^{-4 \pi t\left(L_{0}-c / 24\right)}\right) .
$$

The non-zero mode contribution again agrees with the usual result, while the zero modes contribute a phase due to the additional factor of $i$ in the Gaussian integral. Thus for a single boson we get

$$
Z_{\Omega}^{\mathcal{K}}=\frac{e^{-i \pi / 4}}{\sqrt{\alpha^{\prime} t}} \frac{1}{\eta(2 i t)} .
$$

The Klein Bottle amplitude for the orientifold projection $\Omega I_{1}$ does not receive any zero mode contribution so that one obtains

$$
Z_{\Omega I_{1}}^{\mathcal{K}}=\operatorname{Tr}\left(\Omega I_{1} q^{L_{0}-c / 24} \bar{q}^{L_{0}-c / 24}\right)=e^{\frac{i \pi}{24}} \sqrt{2} \sqrt{\frac{\eta(2 i t)}{\theta_{2}(2 i t)}} .
$$

Turning to the open string sector, the action of the orientifold on the modes is

$$
\Omega \alpha_{n}^{\mu} \Omega^{-1}= \pm(-1)^{n} \alpha_{n}^{\mu},
$$

with the plus sign for NN boundary conditions and the minus sign for DD conditions. Again the non-zero modes agree with the usual expressions. As in the DD sector there is no zero mode contribution in the open string channel, the Möbius strip amplitude is as usual

$$
Z^{\mathcal{M}(D D)}=e^{\frac{i \pi}{24}} \sqrt{2} \sqrt{\frac{\eta\left(i t+\frac{1}{2}\right)}{\theta_{2}\left(i t+\frac{1}{2}\right)}} .
$$

The NN amplitude receives an additional phase from the Gaussian integral so that

$$
Z^{\mathcal{M}(N N)}=e^{\frac{i \pi}{24}} \frac{e^{-i \pi / 4}}{\sqrt{2 \alpha^{\prime} t}} \frac{1}{\eta\left(i t+\frac{1}{2}\right)} .
$$

Therefore, both the former annulus amplitudes and these additional non-oriented one-loop amplitudes differ from the usual ones for Lorentzian signature by the same relative phases. The next step is to introduce the corresponding crosscap states satisfying the usual crosscap gluing conditions and allowing the description of the amplitudes in tree-channel. Moreover, 
one can add the contributions from the world-sheet fermions. However, also here the only difference to the standard case is the appearance of the same phases as already experienced for the D-brane boundary states. Thus, we refrain from presenting the explicit form.

Performing now a full orientifold projection ${ }^{12} \Omega I_{9-p}$ of the Euclidean type IIA/B superstring theories, the tadpole cancellation conditions go through as usual, the Op-planes will have tension

$$
T_{O p}=-2^{p-4} T_{D p}
$$

Introducing time-like directions has the same effect on the phase of the tension as for the corresponding boundary states. To cancel the tadpole induced by the orientifold projection one can introduce stacks of Dp-branes on top of the orientifold planes.

\section{D-branes for exotic string theories}

In this section we further investigate D-branes in the exotic string theories. The main question is which of these branes carry a ghost-free low-energy effective action. Here we will not analyze the full action for an in general intersecting brane system, but as a first step we will restrict to the kinetic term of the gauge field itself.

As mentioned previously, the motivation behind this analysis is that, being agnostic about the fate and meaning of ghosts in gravity (closed string) theories, the experimentally accessible gauge theory (open string) sector should satisfy the usual requirements that we impose on quantum field theories like the Standard Model, namely freedom from physical ghosts and unitarity. Indeed, should we find a ghost-free gauge sector in a theory with closed string ghosts, our pragmatic approach would open a window for dS in string theory.

\subsection{D-branes for Euclidean exotic strings}

First, we consider the Euclidean exotic string theories studied in section 4 and their Dbranes. Having already constructed the corresponding boundary states in a CFT approach, we will now determine their effective action by applying the map (2.19) derived from the negative brane scenario to the DBI action of the D-branes in type IIA/B superstrings. Note that the negative tension $\widehat{\mathrm{Dp}}$-brane should be considered just as a nice tool to identify the correct map from regular to exotic theories as in (2.24). In the following, we will call this brane the defining $\widehat{D p}$-brane.

Exotic DBI actions. The usual DBI+CS action for a Dq-brane (in IIA/ $\mathrm{IB}^{++}$string theories) can be expanded as:

$$
\begin{aligned}
S_{\mathrm{DBI}+\mathrm{CS}}= & -T_{q} \int_{\Sigma_{q+1}} \mathrm{~d}^{q+1} x \sqrt{|g|} e^{-\phi}\left[1+\frac{1}{4}\left(2 \pi \alpha^{\prime}\right)^{2} F_{\mu \nu} F^{\mu \nu}+\ldots\right] \\
& +\mu_{q} \int_{\Sigma_{q+1}}\left[C_{q+1}+F \wedge C_{q-1}+\ldots\right]
\end{aligned}
$$

where $F$ denotes the gauge field strength on the brane and $C_{p}$ bulk R-R p-forms. Of course, for a BPS Dq-brane the tension is the same as the R-R charge, i.e. $T_{q}=\mu_{q}>0$.

\footnotetext{
${ }^{12} \mathrm{As}$ already shown in figure 3 there will be extra factors of $(-1)^{F_{L}}$ in certain cases.
} 
The above action is ghost-free, since the gauge kinetic term has the expected overall minus sign. When one performs the mapping to the exotic string theories, there are two places in the above action where factors of $i$ (or signs) will arise. The first is the relative sign between the two terms in the DBI part: since $|F|^{2}$ contains two inverse metric factors, it is clear that under (2.19) it will pick up a minus factor. This relative sign change happens always, regardless of the number of dimensions that change signature or the dimension of the Dq-brane. The second place is the overall sign of the DBI part due to the rescaling of the dilaton as well as the rescaling of the measure. The factor coming from the dilaton depends on the number $p$ of space-time dimensions that change sign, while the rescaling of the measure now depends on the position (number of parallel and transverse dimensions $n_{\|}$, $n_{\perp}$ ) of the Dq-brane in the signature-changing space-time directions. Since the topological CS term does not contain dilaton or metric factors, the only change there can come from the transformation of the R-R form $C_{q+1}$ which we haven't fully determined here (see also footnote 11).

It is worth noting that even though we are dealing with factors of $i$, all of them nicely cancel out for BPS configurations, giving at most an overall sign change. Here BPS means that the Dq-brane is supersymmetric relative to the defining $\widehat{D p}$-brane. The requirement for a Dq-brane to be BPS can be translated to the condition

$$
n_{\perp}+(p+1)-n_{\|}=0 \bmod 4
$$

As a consequence, the DBI action for a BPS Dq-brane in the exotic theory can only take one of the two forms

$$
S_{\mathrm{DBI}}=\left\{\begin{array}{l}
-T_{q} \int \mathrm{d}^{q+1} x \sqrt{|g|} e^{-\phi}\left[1-\frac{1}{4}\left(2 \pi \alpha^{\prime}\right)^{2} F_{\mu \nu} F^{\mu \nu}+\ldots\right] \pm \mu_{q} \int\left[C_{q+1}+\ldots\right] \\
+T_{q} \int \mathrm{d}^{q+1} x \sqrt{|g|} e^{-\phi}\left[1-\frac{1}{4}\left(2 \pi \alpha^{\prime}\right)^{2} F_{\mu \nu} F^{\mu \nu}+\ldots\right] \pm \mu_{q} \int\left[C_{q+1}+\ldots\right]
\end{array}\right.
$$

Note that the relative sign in front of the kinetic term of the gauge field changed in both cases. We believe that this reflects the generic sign change reported in [36] for all D-branes in Euclidean exotic string theories. In addition, the methods employed in this paper also allow us to determine the sign of the overall normalization (tension).

In the upper case the overall sign is the usual minus. The action is of the same form as the usual DBI+CS action, with the significant difference that the sign in front of the gauge kinetic term is now altered. Hence, the gauge field comes with a kinetic term of the wrong overall sign so that this brane sector is not ghost-free. The physical interpretation of the second action is also clear. The gauge kinetic term comes with the usual negative sign, so the gauge sector is ghost-free. However, the first term in the bracket now carries a relative negative sign with respect to the usual case. Therefore, such an exotic Dq-brane has negative tension.

Classification of BPS branes. We will now move forward and present a comprehensive classification of the BPS branes that appear in the Euclidean exotic string theories. This will enable us to verify the involved CFT construction from the previous section. 
We start with the regular type IIB theory and consider a defining $\widehat{D p}$-brane (with $\mathrm{p}$ odd) that is Lorentzian. Then the map (2.19) gives the exotic Euclidean IIB theory

$$
\operatorname{IIB}_{(9,1)}^{++} \longrightarrow \operatorname{IIB}_{(10-\mathrm{p}, \mathrm{p})}^{-(-)^{\frac{\mathrm{p}-1}{2}}}
$$

and the corresponding mirror theories (2.18). Next, we introduce all possible relatively BPS, Lorentzian Dq-branes in the original type IIB theory and map them via (2.19) to the corresponding Dq-brane in the exotic $\operatorname{IIB}_{(10-\mathrm{p}, \mathrm{p})}^{-(-)^{(\mathrm{p}-1) / 2}}$ theory. ${ }^{13}$ Hence, $(p+1)$ is the number of space-time directions $x_{i}$ which will change signature, while the signature of the other $(9-p)$ directions $y_{j}$ stays the same.

Let us mention again that we denote by $n_{\|}$the number of dimensions along the signature changing $x_{i}$ 's, and $n_{\perp}$ the number of dimensions along the $y_{i}$ 's, with $n_{\|}+n_{\perp}=q+1$. A Dq-brane in the exotic string theory is denoted as $\operatorname{Dq}_{(10-p, p)}^{(s, t)}$, where the pair $(s, t)$ adds up to $q+1$ and indicates the signature of the brane.

Then applying the map to the metric $g$ on Dq, the measure picks up a factor of $\omega^{-1 / 2}$ for each signature changing direction, and a factor of $\omega^{1 / 2}$ for the others. The metric and the dilaton transform exactly as in (2.19). It is then straightforward to determine how the DBI action for the Dq-brane transforms

$$
\begin{aligned}
S_{\mathrm{DBI}} & \rightarrow-T_{q} \int \mathrm{d}^{q+1} x \sqrt{|g|} \omega^{\frac{n_{\perp}-n_{\|}}{2}} \omega^{\frac{p-3}{2}} e^{-\phi}\left[1-\frac{1}{4}\left(2 \pi \alpha^{\prime}\right)^{2} F_{\mu \nu} F^{\mu \nu}+\ldots\right] \\
& =-\omega^{\frac{p+n_{\perp}-n_{\|}-3}{2}} T_{q} \int \mathrm{d}^{q+1} x \sqrt{|g|} e^{-\phi}\left[1-\frac{1}{4}\left(2 \pi \alpha^{\prime}\right)^{2} F_{\mu \nu} F^{\mu \nu}+\ldots\right],
\end{aligned}
$$

which allows to read-off the tension of the brane in the exotic string theory. We note that depending on the position of the Dq-brane, we might get branes of the same dimension which nevertheless have different tensions. As long as all branes of the same dimension are either Lorentzian or Euclidean, it is still consistent with our general framework. Conveniently, this will be satisfied automatically.

Example with defining $\widehat{\text { D3 }}$-brane. Let us present an illustrative example. We pick a defining $\widehat{\mathrm{D} 3}$-brane and present all the consistent branes in the corresponding exotic theories, namely $\operatorname{IIB}_{(7,3)}^{--}$and its "mirror" $\operatorname{IIB}_{(3,7)}^{--}$. For $p=3$, either the first four directions $\left(x^{i}, i=\right.$ $0, \ldots, 3)$ will get their signature reversed, leading to a $(7,3)$ space-time, or the six last $\left(x^{i}, i=4, \ldots, 9\right)$, leading to a $(3,7)$ space-time. Then we successively consider D1-, D3-, ., D9-branes and put them in relatively supersymmetric positions to the defining $\widehat{\mathrm{D} 3}$-brane.

For example, one can see from table 4 that for the D1-brane there exists essentially only one possibility. In the "brane positioning" column of the table, we denote by the superscript whether a direction is space-like $(\mathrm{s})$ or time-like $(\mathrm{t})$ in the $(7,3)$ theory. The subscript denotes the same for the "mirror" $(3,7)$ theory. One can read off that the type IIB D1-brane maps to a Euclidean D1 $(7,3)$-brane with positive tension in the exotic $(7,3)$

\footnotetext{
${ }^{13}$ While we will use a notation inspired by the negative brane horizon crossing of section 2.2 , let us stress once again that we only utilize a negative brane in an intermediate step but are finally interested in the result of mapping a usual BPS type IIB Dq-brane to the exotic string theories.
} 


\begin{tabular}{|c|c|c|c|c|c|c|c|c|c|c|c|c|c|c|c|c|c|}
\hline \multirow{2}{*}{$\mathrm{Dq}$} & \multirow{2}{*}{$\mathrm{n}_{\perp}$} & \multirow{2}{*}{$\mathrm{n}_{\|}$} & \multirow{2}{*}{\multicolumn{2}{|c|}{$\mathrm{k}$ Tension }} & \multicolumn{10}{|c|}{ Brane positioning } & \multirow{2}{*}{$\begin{array}{c}\operatorname{IIB}_{(7,3)}^{--} \\
\text {Brane }\end{array}$} & \multirow{2}{*}{$\begin{array}{l}\operatorname{IIB}_{(3,7)}^{--} \\
\text {Brane }\end{array}$} & \multirow{2}{*}{$\begin{array}{c}\text { Brane } \\
\text { Type(E/L) }\end{array}$} \\
\hline & & & & & $0_{t}^{s}$ & $1_{s}^{t}$ & $2_{s}^{t}$ & $3_{s}^{t}$ & $4_{t}^{s}$ & $5_{t}^{s}$ & $6_{t}^{s}$ & $7_{t}^{s}$ & $8_{t}^{s}$ & $9_{t}^{s}$ & & & \\
\hline D1 & 1 & 1 & 1 & + & $\checkmark$ & - & - & - & $\checkmark$ & - & - & - & - & - & $\mathrm{D} 1_{(7,3)}^{(2,0)}$ & $\mathrm{D} 1_{(3,7)}^{(0,2)}$ & $\mathrm{E}$ \\
\hline D3 & 0 & 4 & 0 & - & $\checkmark$ & $\checkmark$ & $\checkmark$ & $\checkmark$ & - & - & - & - & - & - & $\mathrm{D} 3_{(7,3)}^{(1,3)}$ & $\mathrm{D} 3_{(3,7)}^{(3,1)}$ & $\mathrm{L}$ \\
\hline D3 & 2 & 2 & 1 & + & $\checkmark$ & $\checkmark$ & - & - & $\checkmark$ & $\checkmark$ & - & - & - & - & $\mathrm{D} 3_{(7,3)}^{(3,1)}$ & $\mathrm{D} 3_{(3,7)}^{(1,3)}$ & $\mathrm{L}$ \\
\hline D5 & 3 & 3 & 1 & + & $\checkmark$ & $\checkmark$ & $\checkmark$ & - & $\checkmark$ & $\checkmark$ & $\checkmark$ & - & - & - & $\mathrm{D} 5_{(7,3)}^{(4,2)}$ & $\mathrm{D} 5_{(3,7)}^{(2,4)}$ & $\mathrm{E}$ \\
\hline D5 & 5 & 1 & 2 & - & $\checkmark$ & - & - & - & $\checkmark$ & $\checkmark$ & $\checkmark$ & $\checkmark$ & $\checkmark$ & - & $\mathrm{D} 5_{(7,3)}^{(6,0)}$ & $\mathrm{D} 5_{(3,7)}^{(0,6)}$ & $\mathrm{E}$ \\
\hline D7 & 4 & 4 & 1 & + & $\checkmark$ & $\checkmark$ & $\checkmark$ & $\checkmark$ & $\checkmark$ & $\checkmark$ & $\checkmark$ & $\checkmark$ & - & - & $\mathrm{D} 7_{(7,3)}^{(5,3)}$ & $\mathrm{D} 7_{(3,7)}^{(3,5)}$ & $\mathrm{L}$ \\
\hline D7 & 2 & 6 & 2 & - & $\checkmark$ & $\checkmark$ & - & - & $\checkmark$ & $\checkmark$ & $\checkmark$ & $\checkmark$ & $\checkmark$ & $\checkmark$ & $\mathrm{D} 7_{(7,3)}^{(7,1)}$ & $\mathrm{D} 7_{(3,7)}^{(1,7)}$ & $\mathrm{L}$ \\
\hline D9 & & & & o consist & tent & $D 9-$ & -bran & ne c & confi & gur & ation & & & & - & - & - \\
\hline
\end{tabular}

Table 4. Brane spectrum of $\operatorname{IIB}_{(7,3) /(3,7)}^{--}$theories.

theory. Being Euclidean, this is consistent with a $\mathrm{IIB}^{--}$theory. Moreover, being of positive tension implies that the gauge field on the brane is a ghost field.

Similarly, one can analyze the higher dimensional Dq-branes and fill out the entire table 4. As expected, the signatures (Lorentzian/Euclidean) of the branes alternate. There are 7 different BPS configurations allowed. Out of these, 3 have negative tension and are therefore ghost-free. We should note here that the table only includes the overall sign of the brane tension, as the precise value is irrelevant for the present discussion. Let us also stress that while there exist negative tension (ghost-free) D3-, D5- and D7- branes, not all are of this type since the arrangement of the branes in space-time plays a crucial role. We observe that the brane spectrum of table 4 could be partially incomplete, since additional branes can occur with the mirror dual $p=7$ mapping.

We can now perform the classification also for $p=1,5,7,9$. In table 8 of appendix $\mathrm{B}$ we present the brane spectrum of $\operatorname{IIB}_{(9,1)}^{-+}$and its mirror dual $\mathrm{IIB}_{(1,9)}^{-+}$. In table 9 we give the result for $\operatorname{IIB}_{(7,3) /(3,7)}^{--}$and in table 10 that for $\operatorname{IIB}_{(5,5) /(5,5)}^{-+}$. In a similar fashion, we computed the D-brane spectrum of the exotic Euclidean type IIA theories in the various consistent signatures. We present the results in tables 11, 12, 13 of appendix C. Note that there is one major difference to the type IIB case: while for type IIB the space-time mirror theories are of the same type, in type IIA the space-time mirror also affects the type of the theory, in particular whether the branes are Euclidean or Lorentzian. The Euclidean type IIA space-time mirrors are $\operatorname{IIA}_{(10-p, p)}^{--} \leftrightarrow \operatorname{IIA}_{(p, 10-p)}^{-+}$. Finally, we want to stress that the results of the present section 5 are in complete accord with the brane spectrum of section 4 , which was acquired using Euclidean CFT techniques. Our results are also compatible with the spectra reviewed in tables 1 and 2. In addition we found the missing D8 and D9 branes, and more importantly derived the signs of the tensions for all the branes. 


\subsection{Ghost-free D-brane theories}

Scanning through the tables we extract all D-branes that are ghost-free (i.e. have negative tension) and contain a $(3,1)$ subspace. These are the D-branes that have a chance to lead to a viable phenomenology. These ghost-free branes are

$$
\begin{aligned}
& \text { type IIB : } \quad \mathrm{D} 9_{(9,1)}^{(9,1)}, \quad \mathrm{D} 7_{(7,3)}^{(7,1)}, \quad \mathrm{D} 5_{(5,5)}^{(5,1)}, \quad \mathrm{D} 3_{(3,7)}^{(3,1)} \text {, } \\
& \text { type IIA : } \quad \mathrm{D} 8_{(8,2)}^{(8,1)}, \quad \mathrm{D} 6_{(6,4)}^{(6,1)}, \quad \mathrm{D} 4_{(4,6)}^{(4,1)} .
\end{aligned}
$$

In the following, we discuss this class of branes in more detail, as they share a couple of common features.

First, all these branes have in common that they are space-filling, but localized in the extra time-like directions. For instance, as can be seen from table 4 , the $\mathrm{D} 7_{(7,3)}^{(7,1)}$ brane is localized in the $t_{2}$ and $t_{3}$ directions and longitudinal along $s_{0}, t_{1}, s_{4}, \ldots, s_{9}$. Compactifying the extra time-like directions and all space-like directions except the three large ones that are to make our world, an open string ending on the brane will have KK modes along the compact space-like directions and winding modes in the compact time-like directions. As a consequence, employing (3.13) the mass spectrum of such an open string reads

$$
E^{2}=\sum_{i}\left(p^{i}\right)^{2}+\sum_{s}\left(\frac{m_{s}}{R_{s}}\right)^{2}+\sum_{t}\left(\frac{n_{t} R_{t}}{\alpha^{\prime}}\right)^{2}-\frac{i}{\alpha^{\prime}}(N-a),
$$

where the indices $s(t)$ indicate space(time)-like directions. Therefore, for these particular branes both KK and winding modes contribute positively to the right hand side of (5.7). This is the same behavior as for D-branes in the usual type IIA/IIB theories. This implies that in contrast to closed strings, for such D-branes there is no issue with an infinite number of open string modes becoming arbitrarily light.

Being localized in the extra time-like directions, the transversal deformations of the D-branes in (5.6) will be ghosts. On a torus such deformations will exist but on a more general background they can be absent, if the brane wraps a rigid cycle. There will certainly exist massive open string ghosts, but they are expected to kinematically decouple from the massless open string states below a cut-off $\Lambda_{\mathrm{UV}}$. Whether also the ultra-light closed string states decouple is a more intricate question. Since they couple gravitationally, they are expected to decouple in the large Planck-mass limit. However, there are in principle infinitely many such states, so it is not a trivial question whether they will have a negligible overall effect on the low-energy scattering of massless open string modes.

The second common feature of the ghost-free D-branes in (5.6) is that they are all directly related to the orientifold projections discussed in section 3.2 and summarized in figure 3. If transversal directions of a D-brane are compactified, its $\mathrm{R}-\mathrm{R}$ charge has to be cancelled, a feature known as tadpole cancellation. Therefore, one is forced to introduce also oppositely charged objects in the backgrounds. These are the orientifold planes constructed in section 4.5. They arise by performing an orientifold projection $\Omega I_{\perp}$, where $I_{\perp}$ reflects the coordinates transversal to the brane. As an example consider $\mathrm{D} 7_{(7,3)}^{(7,1)}$, whose related orientifold quotient is $\operatorname{IIB}_{(7,3)}^{--} / \Omega I_{2}(-1)^{F_{L}}$, where $I_{2}$ reflects the two extra 
time-like coordinates. However, this is precisely the orientifold projection that removes all the 10D massless ghosts in the closed string sector. The same behavior arises for all the ghost-free branes in (5.6). Summarizing, the required orientifold projection that allows us to introduce these branes in the given background in the first place, is also the orientifold that we encountered in figure 3 which projects out all the massless ghosts appearing in the exotic 10D supergravity actions.

Recall now that the de Sitter solutions of the type presented in section 2.1 were only possible because of the existence of massless ghosts in 10D closed string action. Without this loop-hole in the classical dS no-go theorem, dS vacua will very likely not be possible. Therefore, for the Euclidean exotic string theories with in general multiple times, there is a strong correlation between the presence of a phenomenologically viable D-brane (gauge theory) sector and the existence of dS solutions:

Conjecture. A compactified Euclidean exotic string theory contains a $4 D$ ghost-free gauge theory with signature $(3,1)$, iff the closed string sector does not admit classical dS vacua.

This can be interpreted as an extension of the (classical) dS swampland conjecture to Euclidean exotic superstring theories. We still consider it a conjecture, as in principle there could be other orientifold projections still admitting dS solutions, whose tadpole could be cancelled by introducing more involved configurations of intersecting D-branes. The latter will likely contain besides the ghost-free branes (5.6) also some other ones with ghosts. Moreover, there will be additional massless states on the intersection of branes that could also be ghosts. Whether such configurations could lead to a viable ghost-free standard-model subsector remains to be seen, though we doubt it.

\subsection{Orientifolds of Lorentzian exotic strings}

Let us now consider the Lorentzian exotic string theories and analyze whether ghost-free D-branes can be introduced there. Here the $\mathbb{Z}_{2}$ projections from figure 2 that project out the massless $10 \mathrm{D}$ ghosts are not orientifold projections but just $\mathbb{Z}_{2}$ orbifolds.

Thus, first we investigate what kind of $\mathbb{Z}_{2}$ projections the various kinds of such theories do admit. In the second part we will analyze whether there exist orientifolds that support D-branes with a ghost-free kinetic term for the gauge field while still potentially admitting de Sitter solutions in the closed string sector.

Generalities on $\mathbb{Z}_{\mathbf{2}}$ projections. In the following we will be interested in $\Omega I_{m, n}$ orientifolds for the type IIA $/ \mathrm{B}_{(10-p, p)}^{(+, \beta)}$ theories, where $I_{m, n}$ denotes the reflection of $m$ space-like and $n$ time-like directions. As is known already for the usual type II theories there appears a subtlety in the Ramond sector of the theory.

For all theories of signature $(q, p) \in\{(9,1),(5,5),(1,9)\}$ the Clifford algebra

$$
\left\{\Gamma^{A}, \Gamma^{B}\right\}=2 \eta^{A B},
$$

has similar properties (as $q-p=0 \bmod 8$ ). Here, $\eta^{A B}= \pm 1$ for space/time-like directions. Let us recall some of the salient properties of the $\Gamma$-matrices. In the following $\Gamma^{a}$ denote 


\begin{tabular}{|cc|}
\hline$I_{m, n}$ & action on spinor \\
\hline$m$ even, $n$ even & $S^{ \pm} \rightarrow S^{ \pm}$ \\
$m$ odd, $n$ odd & $S^{ \pm} \rightarrow i S^{ \pm}$ \\
\hline$m$ odd, $n$ even & $S^{ \pm} \rightarrow S^{\mp}$ \\
$m$ even, $n$ odd & $S^{ \pm} \rightarrow i S^{\mp}$ \\
\hline
\end{tabular}

Table 5. Action of $I_{m+n}$ reflection on spinors.

space-like directions and $\Gamma^{\alpha}$ time-like ones. All $\Gamma$-matrices are unitary, if they satisfy the following hermiticity conditions

$$
\left(\Gamma^{a}\right)^{\dagger}=\Gamma^{a}, \quad\left(\Gamma^{\alpha}\right)^{\dagger}=-\Gamma^{\alpha}
$$

Moreover, one can define the chirality operator

$$
\Gamma^{10}=\prod_{A} \Gamma^{A}
$$

which anti-commutes with all $\Gamma^{A}$, is Hermitian $\left(\Gamma^{10}\right)^{\dagger}=\Gamma^{10}$ and satisfies $\left(\Gamma^{10}\right)^{2}=1$. One can choose the $\Gamma$-matrices to be purely imaginary in which case $\Gamma^{10}$ is real. In this representation, a Majorana spinor is real.

The Ramond ground state in both the left and the right-moving sector is a MajoranaWeyl spinor in 10D, thus it is chiral and real. Type IIB has two spinors of the same chirality and type IIA two spinors of opposite chirality. These spinors of positive and negative chirality are denoted as usual by $S^{+}$and $S^{-}$.

The reflection along a single space-like direction $x^{a}$ acts on the spinors as

$$
I^{a}: S \rightarrow i \Gamma^{10} \Gamma^{a} S
$$

guaranteeing $\left\{I^{a}, \Gamma^{a}\right\}=0$ and $\left[I^{a}, \Gamma^{B}\right]=0($ for $a \neq B)$. This operation is Hermitian, real and changes the chirality of the spinor. Moreover, it satisfies $\left(I^{a}\right)^{2}=1$. The reflection along a time-like direction can also be chosen to be Hermitian but then it becomes purely imaginary

$$
I^{\alpha}: S \rightarrow \Gamma^{10} \Gamma^{\alpha} S
$$

Thus, $I^{\alpha}$ is Hermitian, imaginary, changes the chirality and satisfies $\left(I^{\alpha}\right)^{2}=1$. Let us now consider a general $\mathbb{Z}_{2}$ reflection

$$
I_{m+n}=\prod_{i=1}^{m} I^{a_{i}} \prod_{j=1}^{n} I^{\alpha_{j}}
$$

along $m$ space-like and $n$ time-like directions. Its action on a spinor is summarized in table 5 . 


\begin{tabular}{|cc|}
\hline Type IIA/B & orientifold \\
\hline Type IIB $^{++}$ & $\Omega I_{2 m, 2 n}\left[(-1)^{F_{L}}\right]^{m+n}$ \\
Type IIB $^{+-}$ & $\Omega I_{2 m-1,2 n-1}\left[(-1)^{F_{L}}\right]^{m+n-1}$ \\
Type IIA $^{++}$ & $\Omega I_{2 m-1,2 n}\left[(-1)^{F_{L}}\right]^{m+n-1}$ \\
Type IIA $^{+-}$ & $\Omega I_{2 m, 2 n-1}\left[(-1)^{F_{L}}\right]^{m+n-1}$ \\
\hline
\end{tabular}

Table 6. Admissible orientifold projections.

Using that $\left\{I^{A}, I^{B}\right\}=2 \delta^{A B}$, one can show that for $m+n=2 k$ or $m+n=2 k+1$ the square of $I_{m+n}$ on the Ramond ground state is $I_{m+n}^{2}=(-1)^{k}$. We are interested in the consistent orientifold projections of type $\Omega I_{m+n}$ for the type IIB/IIA ${ }^{+, \beta}$ string theories. Requiring that the full orientifold projection squares to +1 one obtains the admissible possibilities listed in table 6 for the type IIB/IIA string. Here, as usual the factor $(-1)^{F_{L}}$ is introduced to compensate for $\left(\Omega I_{m+n}\right)^{2}=-1$.

Ghost-free D-branes and dS. Let us now analyze whether orientifolds of type IIA/B $\mathrm{B}^{+, \beta}$ can support D-branes of at least signature $(3,1)$ and without gauge field ghosts while still admitting dS-type solutions in the closed string sector. The type IIA $/ \mathrm{B}_{(1,9)}^{+, \beta}$ theories can be dismissed right away, as they do not have at least three space-like directions. Moreover, type IIA/B $\mathrm{B}_{(9,1)}^{++}$are just the usual type IIA/B theories for which the dS swampland conjecture is supposed to hold. The type IIA/ $\mathrm{B}_{(9,1)}^{+-}$theories only contain Euclidean D-branes that cannot support a gauge theory in $(3,1)$ dimensions. Thus we are left with the type $\operatorname{IIA} / \mathrm{B}_{(5,5)}^{+, \beta}$ theories.

Let us have a closer look at the type $\operatorname{IIA}_{(5,5)}^{++}$theory. This theory still contains Lorentzian fundamental strings so that the CFT is like the usual type IIA theory, only the signature changes from $(9,1)$ to $(5,5)$. All D-branes have positive tension and the usual sign of the kinetic term for the gauge field. Of course, this implies that the time-like components of the gauge field $A^{\mu}$ are ghosts.

As we have seen, it is the $\mathbb{Z}_{2}$ projection $I_{4}$ or $I_{4}(-1)^{F_{L}}$ reflecting the four extra timelike directions that removes all closed string ghosts from the action. Clearly, this is not an orientifold so that it could well be that e.g. an orientifold $\Omega I_{3}(-1)^{F_{L}}$ with O6-planes and corresponding D6-branes gives a ghost-free gauge theory, while still allowing closed string ghosts.

For concreteness, let us consider a compactification on a six torus $T^{6}$. Let us denote the two compact space- and the four compact time-directions as $\left\{x_{1}, x_{2} ; t_{1}, t_{2}, t_{3}, t_{4}\right\}$. Therefore, we can group the six-coordinates in three pairs $\left\{\left(x_{1}, x_{2}\right),\left(t_{1}, t_{2}\right),\left(t_{3}, t_{4}\right)\right\}$ and choose the orientifold projection to be of type $\Omega I_{1,2}(-1)^{F_{L}}$ reflecting the three coordinates $\left\{x_{2}, t_{2}, t_{4}\right\}$. This leads to an O6-planes parallel to the plane $\left\{x_{1}, t_{1}, t_{3}\right\}$. This is shown in figure 4. The induced tadpole can be cancelled by D6-branes on top of the O6-planes. Note that these D6-branes are Lorentzian in the sense that there is an odd number (namely three) of longitudinal time-like directions. 

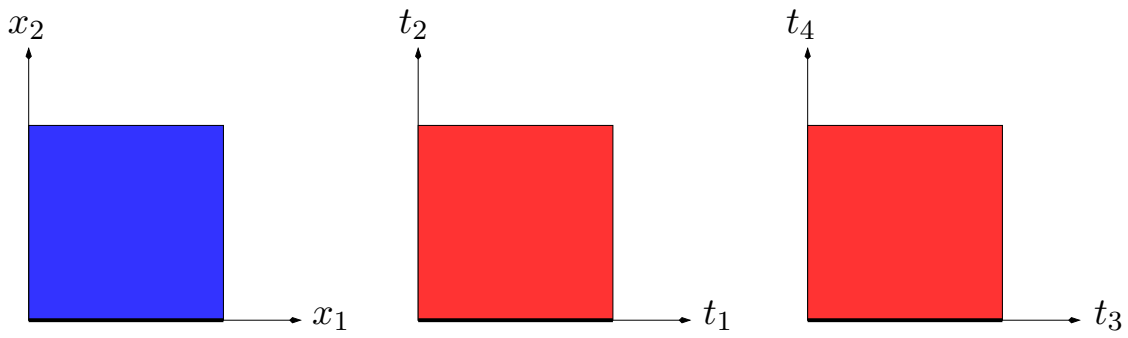

Figure 4. The six-torus of signature $(2,4)$ with O6-plane along $\left(x_{1}, t_{1}, t_{3}\right)$.

\begin{tabular}{|cc|}
\hline Flux & Cohomology \\
\hline$H$ & $H_{-}^{3}(X)$ \\
$\left\{F_{0}, F_{2}, F_{4}, F_{6}\right\}$ & $\left\{H_{+}^{0}, H_{-}^{2}, H_{+}^{4}, H_{-}^{6}\right\}$ \\
\hline
\end{tabular}

Table 7. Equivariant cohomology groups of orientifold even fluxes.

Moreover, the 4D gauge field on these D6-branes has the usual kinetic term and is ghost-free. However, in this toroidal example the Wilson-lines along the $\left\{t_{1}, t_{3}\right\}$ directions and the deformations of the brane in the $\left\{t_{2}, t_{4}\right\}$ directions will be ghosts in the effective 4D theory. However, for more general internal spaces (something like a CY of signature $(2,4))$ these open string moduli could be avoided if the D6-branes wrap a rigid 3-cycle.

In order to see whether dS vacua are in principle possible, let us investigate which flux components survive the orientifold projection. For this purpose we recall the general result about the cohomological classification of the orientifold even fluxes shown in table 7 .

Let us look at $F_{2}$, for which the flux

$$
F_{2}=f d x_{1} \wedge d t_{2}
$$

is in $H_{-}^{2}$ and therefore survives the orientifold projection. Now, since $F_{2}$ is supported along one space-like and one time-like leg, the kinetic term of this two-form flux

$$
\left|F_{2}\right|^{2} \sim g^{i_{1} i_{2}} g^{j_{1} j_{2}}\left(F_{2}\right)_{i_{1} j_{1}}\left(F_{2}\right)_{i_{2} j_{2}}
$$

has the opposite sign to the usual one. For the other fluxes one finds similar ghost-like components, as well. Therefore, this type IIA model features fluxes with the wrong sign of their kinetic terms and thus the usual dS no-go theorem does not apply and dS vacua might be possible. Since we will see below that these exotic orientifolds have other problems, it is beyond the scope of this paper to work out in detail a dS model on a fully fledged "CY" space. At least we can state that up to this point there is no immediate obstacle for dS solutions with a ghost-free massless gauge field on the brane.

Ultralight open string modes. Recall that for the ghost-free D-branes in Euclidean exotic theories, the KK and winding modes were such that they contributed like a positive mass squared $m^{2}$ to the right hand side of the on-shell relation (5.7). This is different for the D-branes in Lorentzian exotic theories. For such a D-brane the on-shell condition now 
reads

$$
\begin{aligned}
E^{2} & +\sum_{t_{\|}}\left(\frac{m_{t}}{R_{t}}\right)^{2}+\sum_{t_{\perp}}\left(\frac{n_{t} R_{t}}{\alpha^{\prime}}\right)^{2} \\
& =\sum_{i}\left(p^{i}\right)^{2}+\sum_{s_{\|}}\left(\frac{m_{s}}{R_{s}}\right)^{2}+\sum_{s_{\perp}}\left(\frac{n_{s} R_{s}}{\alpha^{\prime}}\right)^{2}+\frac{1}{\alpha^{\prime}}(N-a)
\end{aligned}
$$

where the indices $(s / t)_{\|}$and $(s / t)_{\perp}$ indicate space-/time-like directions parallel and perpendicular to the D-brane world-volume. Therefore, here both time-like KK and time-like winding modes contribute always to the left hand side of this relation. As for the closed string, these time-like modes can cancel against oscillatory modes yielding infinitely many arbitrarily light open string modes. This questions the role of the Wilsonian effective gauge theory action on the D-brane and seems to be a general problem with a potential phenomenological application of orientifolds of Lorentzian exotic superstring theories (with multiple times).

\section{Conclusions}

In this paper we performed a detailed study of the brane sector in exotic string theories. Driven by the motivating example of dS solutions in supergravity theories with more than one time direction, we investigated the behavior of exotic string theories upon torus compactification. Despite being able to get rid of massless ghosts in the 10D theory by performing suitable orientifold projections, we found that torus compactifications with time-like circles lead to an unsatisfactory infinite tower of arbitrarily light states in the lower dimensional effective theories. One can ask if this is a generic behavior of timelike compactifications. In particular there exist pseudo-Riemannian analogs of Calabi-Yau manifolds with reduced holonomy (see the article of $\mathrm{H}$. Baum in [55]) which would partially preserve supersymmetry upon compactifying exotic string theories on them.

Although the closed string sector seems to show unavoidable pathologies, there might still be a phenomenologically consistent brane sector coupling to those "bizarre" quantum gravity theories. After all, experiments today don't test quantum gravity, hence we adopted an agnostic point of view and merely asked for a well behaved massless open string sector. Taking a perturbative point of view (after all, the dS solutions arise for the leading order supergravity actions) we continued along the line of [39] and constructed a CFT description of closed and in particular open Euclidean exotic string theories. Guided by consistency of the mathematical formalism we derived all allowed D-branes (of real tension) in exotic string theories with all possible metric signatures. Notably we identified in every exotic theory a phenomenologically consistent D-brane lacking massless ghosts and having a $(3,1)$ signature subspace.

The same classification of allowed branes was obtained by considering negative tension branes in type IIA/B supergravity theories and performing an analytic continuation of Dbrane actions beyond the horizon in the singular space-time. As a special feature of those branes we found that from a space-time point of view, their existence is related to precisely 
the orientifold projections used to discard massless closed string ghosts. We formulated this result as an "exotic" de Sitter no-go conjecture.

Of course we cannot claim to have given a viable interpretation/description of quantum gravity with multiple time-like directions. While the formalism of conformal field theory and supergravity appears to go through for such theories, their conceptual interpretation still remains elusive and we have not much to add to that. Admittedly, we also left open a couple of important and interesting technical questions, the most pressing of which is the role of supersymmetry. Related to this is the question of general compactifications on manifolds with pseudo-Riemannian metrics that go beyond the toroidal case.

\section{Acknowledgments}

We thank Chris Hull for very useful comments about a former version of this paper. Furthermore we thank Brage Gording for discussions and Ben Heidenreich for useful comments following a talk by RB given at the String Phenomenology 2019 conference at CERN.

\section{A (A)dS spaces of signature $(p, q)$}

First we introduce the notion of anti-de Sitter and de Sitter spaces with signature $(p, q)$ where $p$ denotes the number of time-like directions.

$\boldsymbol{A d} \boldsymbol{S}_{p, q}$ spaces. This space is defined as the real hypersurface

$$
-\sum_{i=0}^{p} t_{i}^{2}+\sum_{j=1}^{q} x_{j}^{2}=-\alpha^{2}
$$

in $\mathbb{R}_{p+1, q}$, where $\alpha$ denotes a real number. A solution to this equation can be written as

$$
\begin{aligned}
t_{0} & =\alpha \cosh (\rho / \alpha)+\frac{e^{\rho / \alpha}}{2 \alpha}\left(-\sum_{i=1}^{p} \hat{t}_{i}^{2}+\sum_{j=2}^{q} \hat{x}_{j}^{2}\right) \\
x_{1} & =\alpha \sinh (\rho / \alpha)-\frac{e^{\rho / \alpha}}{2 \alpha}\left(-\sum_{i=1}^{p} \hat{t}_{i}^{2}+\sum_{j=2}^{q} \hat{x}_{j}^{2}\right) \\
t_{i} & =e^{\rho / \alpha} \hat{t}_{i}, \quad i=1, \ldots p \\
x_{j} & =e^{\rho / \alpha} \hat{x}_{j}, \quad j=2, \ldots q .
\end{aligned}
$$

The metric on $A d S_{p, q}$ is then given as

$$
d s^{2}=d \rho^{2}+e^{2 \rho / \alpha}\left(-\sum_{i=1}^{p} d \hat{t}_{i}^{2}+\sum_{j=2}^{q} d \hat{x}_{j}^{2}\right)
$$

which is the so-called flat slicing of $A d S_{p, q}$. One can introduce a corresponding $(p+q)$-bein $E^{A}$ on $A d S_{p, q}$ so that the metric (A.3) takes the simple form $d s^{2}=\eta_{A B}^{(p, q)} E^{A} E^{B}$. The resulting Ricci-tensor in this frame reads

$$
R_{A B}=-\eta_{A B}^{(p, q)} \frac{(p+q-1)}{\alpha^{2}}
$$

so that the Ricci-scalar becomes $R=-(p+q)(p+q-1) / \alpha^{2}$. 
$\boldsymbol{d} \boldsymbol{S}_{\boldsymbol{p}, \boldsymbol{q}}$ spaces. Similarly, one can define and describe de Sitter spaces with signature $(p, q)$. It is defined as the real hypersurface

$$
-\sum_{i=0}^{p-1} t_{i}^{2}+\sum_{j=1}^{q+1} x_{j}^{2}=\beta^{2}
$$

in $\mathbb{R}_{p, q+1}$ with a solution given as

$$
\begin{aligned}
t_{0} & =\beta \sinh (\tau / \beta)+\frac{e^{\tau / 2 \beta}}{2 \beta}\left(-\sum_{i=1}^{p-1} \hat{t}_{i}^{2}+\sum_{j=2}^{q+1} \hat{x}_{j}^{2}\right) \\
x_{1} & =\beta \cosh (\tau / \beta)-\frac{e^{\tau / 2 \beta}}{2 \beta}\left(-\sum_{i=1}^{p-1} \hat{t}_{i}^{2}+\sum_{j=2}^{q+1} \hat{x}_{j}^{2}\right) \\
t_{i} & =e^{\tau / \beta} \hat{t}_{i}, \quad i=1, \ldots p-1 \\
x_{j} & =e^{\tau / \beta} \hat{x}_{j}, \quad j=2, \ldots q+1
\end{aligned}
$$

yielding the metric

$$
d s^{2}=-d \tau^{2}+e^{2 \tau / \beta}\left(-\sum_{i=1}^{p-1} d \hat{t}_{i}^{2}+\sum_{j=2}^{q+1} d \hat{x}_{j}^{2}\right),
$$

\begin{tabular}{|c|c|c|c|c|c|c|c|c|c|c|c|}
\hline Theory & $\mathrm{Dp}$ & Branes & Type(E/L) & Tension & Ghost-free & Theory & $\mathrm{Dp}$ & Branes & Type(E/L) & Tension & Ghost-free \\
\hline \multirow{6}{*}{$\operatorname{IIB}_{(9,1)}^{-+}$} & $\mathrm{D}(-1)$ & $\mathrm{D}(-1)_{(9,1)}^{(0,0)}$ & $\mathrm{E}$ & - & $\checkmark$ & \multirow{6}{*}{$\operatorname{IIB}_{(1,9)}^{-+}$} & $\mathrm{D}(-1)$ & $\mathrm{D}(-1)_{(1,9)}^{(0,0)}$ & $\mathrm{E}$ & - & $\checkmark$ \\
\hline & D1 & $\mathrm{D} 1_{(9,1)}^{(1,1)}$ & $\mathrm{L}$ & - & $\checkmark$ & & D1 & $\mathrm{D} 1_{(1,9)}^{(1,1)}$ & $\mathrm{L}$ & - & $\checkmark$ \\
\hline & D3 & $\mathrm{D} 3_{(9,1)}^{(4,0)}$ & $\mathrm{E}$ & + & - & & D3 & $\mathrm{D} 3_{(1,9)}^{(0,4)}$ & $\mathrm{E}$ & + & - \\
\hline & D5 & $\mathrm{D} 5_{(9,1)}^{(5,1)}$ & $\mathrm{L}$ & + & - & & D5 & $\mathrm{D} 5_{(1,9)}^{(1,5)}$ & $\mathrm{L}$ & + & - \\
\hline & D7 & $\mathrm{D} 7_{(9,1)}^{(8,0)}$ & $\mathrm{E}$ & - & $\checkmark$ & & D7 & $\mathrm{D} 7_{(1,9)}^{(0,8)}$ & E & - & $\checkmark$ \\
\hline & D9 & $\mathrm{D} 9_{(9,1)}^{(9,1)}$ & L & - & $\checkmark$ & & D9 & $\mathrm{D} 9_{(1,9)}^{(1,9)}$ & $\mathrm{L}$ & - & $\checkmark$ \\
\hline
\end{tabular}

from which one can read of a $(p+q)$-bein $\mathcal{E}_{A}$. The resulting Ricci-tensor reads

$$
R_{A B}=\eta_{A B}^{(p, q)} \frac{(p+q-1)}{\beta^{2}}
$$

with the Ricci-scalar $R=(p+q)(p+q-1) / \beta^{2}$.

\section{B Table of branes in exotic IIB theories}

Table 8. Brane spectrum of mirror $\operatorname{IIB}_{(9,1) /(1,9)}^{-+}$theories. 


\begin{tabular}{|c|c|c|c|c|c|c|c|c|c|c|c|}
\hline Theory & Dp & Branes & Type(E/L) & Tension & Ghost-free & Theory & Dp & Branes & Type(E/L) & Tension & Ghost-free \\
\hline \multirow{8}{*}{$\operatorname{IIB}_{(7,3)}^{--}$} & D1 & $\mathrm{D} 1_{(7,3)}^{(2,0)}$ & $\mathrm{E}$ & + & - & \multirow{8}{*}{$\operatorname{IIB}_{(3,7)}^{--}$} & \multirow[t]{2}{*}{ D1 } & $\mathrm{D} 1_{(3,7)}^{(0,2)}$ & $\mathrm{E}$ & + & - \\
\hline & & $\mathrm{D} 1_{(7,3)}^{(0,2)}$ & $\mathrm{E}$ & - & $\checkmark$ & & & $\mathrm{D} 1_{(3,7)}^{(2,0)}$ & $\mathrm{E}$ & - & $\checkmark$ \\
\hline & D3 & $\mathrm{D} 3_{(7,3)}^{(3,1)}$ & $\mathrm{L}$ & + & - & & \multirow[t]{2}{*}{ D3 } & $\mathrm{D} 3_{(3,7)}^{(1,3)}$ & $\mathrm{L}$ & + & - \\
\hline & & $\mathrm{D} 3_{(7,3)}^{(1,3)}$ & $\mathrm{L}$ & - & $\checkmark$ & & & $\mathrm{D} 3_{(3,7)}^{(3,1)}$ & $\mathrm{L}$ & - & $\checkmark$ \\
\hline & D5 & $\mathrm{D} 5_{(7,3)}^{(4,2)}$ & $\mathrm{E}$ & + & - & & \multirow[t]{2}{*}{ D5 } & $\mathrm{D} 5_{(3,7)}^{(2,4)}$ & $\mathrm{E}$ & + & - \\
\hline & & $\mathrm{D} 5_{(7,3)}^{(6,0)}$ & $\mathrm{E}$ & - & $\checkmark$ & & & $\mathrm{D} 5_{(3,7)}^{(0,6)}$ & $\mathrm{E}$ & - & $\checkmark$ \\
\hline & D7 & $\mathrm{D} 7_{(7,3)}^{(5,3)}$ & $\mathrm{L}$ & + & - & & \multirow[t]{2}{*}{ D7 } & $\mathrm{D} 7_{(3,7)}^{(3,5)}$ & $\mathrm{L}$ & + & - \\
\hline & & $\mathrm{D} 7_{(7,3)}^{(7,1)}$ & $\mathrm{L}$ & - & $\checkmark$ & & & $\mathrm{D} 7_{(3,7)}^{(1,7)}$ & $\mathrm{L}$ & - & $\checkmark$ \\
\hline
\end{tabular}

Table 9. Brane spectrum of mirror $\operatorname{IIB}_{(7,3) /(3,7)}^{--}$theories.

\begin{tabular}{|cccccc|}
\hline Theory & Dp & Branes & Type(E/L) & Tension & Ghost-free \\
\hline & $\mathrm{D}(-1)$ & $\mathrm{D}(-1)_{(5,5)}^{(0,0)}$ & $\mathrm{E}$ & + & - \\
$\mathrm{D} 1$ & $\mathrm{D} 1_{(5,5)}^{(1,1)}$ & $\mathrm{L}$ & + & - \\
& $\mathrm{D} 3$ & $\mathrm{D} 3_{(5,5)}^{(2,2)}$ & $\mathrm{E}$ & + & - \\
& & $\mathrm{D} 3_{(5,5)}^{(0,4)}$ & $\mathrm{E}$ & - & $\checkmark$ \\
$\mathrm{IIB}_{(5,5)}^{-+}$ & & $\mathrm{D} 3_{(5,5)}^{(4,0)}$ & $\mathrm{E}$ & - & $\checkmark$ \\
& $\mathrm{D} 5$ & $\mathrm{D} 5_{(5,5)}^{(3,3)}$ & $\mathrm{L}$ & + & - \\
& & $\mathrm{D} 5_{(5,5)}^{(1,5)}$ & $\mathrm{L}$ & - & $\checkmark$ \\
& $\mathrm{D} 5_{(5,5)}^{(5,1)}$ & $\mathrm{L}$ & - & $\checkmark$ \\
& $\mathrm{D} 7$ & $\mathrm{D} 7_{(5,5)}^{(4,4)}$ & $\mathrm{E}$ & + & - \\
$\mathrm{D} 9$ & $\mathrm{D} 9_{(5,5)}^{(5,5)}$ & $\mathrm{L}$ & + & - \\
\hline
\end{tabular}

Table 10. Brane spectrum of $\operatorname{IIB}_{(5,5)}^{-+}$.

\section{Table of branes in exotic IIA theories}

\begin{tabular}{|c|c|c|c|c|c|c|c|c|c|c|c|}
\hline Theory & Dp & Branes & Type(E/L) & Tension & Ghost-free & Theory & Dp & Branes & Type(E/L) & Tension & Ghost-free \\
\hline \multirow{5}{*}{$\operatorname{IIA}_{(10,0)}^{-+}$} & D0 & $\mathrm{D} 0_{(10,0)}^{(1,0)}$ & $\mathrm{E}$ & - & $\checkmark$ & \multirow{5}{*}{$\operatorname{IIA}_{(0,10)}^{--}$} & D0 & $\mathrm{D} 0_{(0,10)}^{(0,1)}$ & $\mathrm{L}$ & - & $\checkmark$ \\
\hline & D2 & No co & nsistent D2- $t$ & rane con & iguration & & D2 & \multicolumn{4}{|c|}{ No consistent D2-brane configuration } \\
\hline & $\mathrm{D} 4$ & $\mathrm{D} 4_{(10,0)}^{(5,0)}$ & $\mathrm{E}$ & + & - & & $\mathrm{D} 4$ & $\mathrm{D} 4_{(0,10)}^{(0,5)}$ & $\mathrm{L}$ & + & - \\
\hline & D6 & No co & nsistent $D 6-t$ & rane con & iguration & & D6 & \multicolumn{4}{|c|}{ No consistent D6-brane configuration } \\
\hline & D8 & $\mathrm{D} 8_{(10,0)}^{(9,0)}$ & $\mathrm{E}$ & - & $\checkmark$ & & D8 & $\mathrm{D} 8_{(0,10)}^{(0,9)}$ & $\mathrm{L}$ & - & $\checkmark$ \\
\hline
\end{tabular}

Table 11. Brane spectrum of mirror $\operatorname{IIA}_{(10,0)}^{-+}$and $\operatorname{IIA}_{(0,10)}^{--}$theories. 


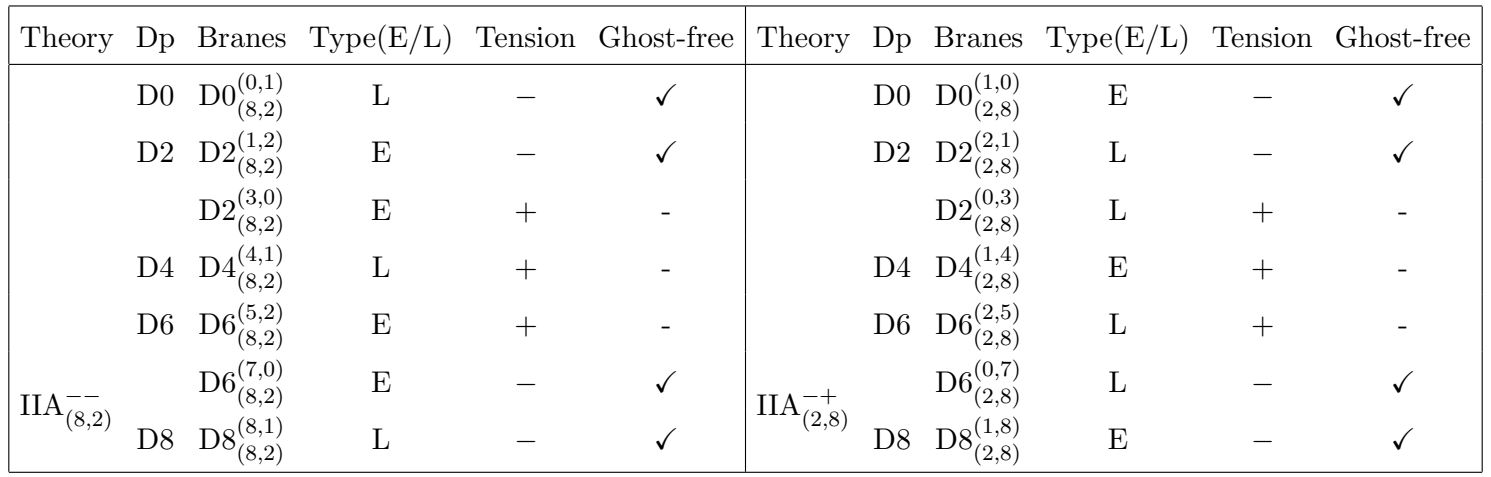

Table 12. Brane spectrum of mirror $\operatorname{IIA}_{(8,2)}^{--}$and $\operatorname{IIA}_{(2,8)}^{-+}$theories.

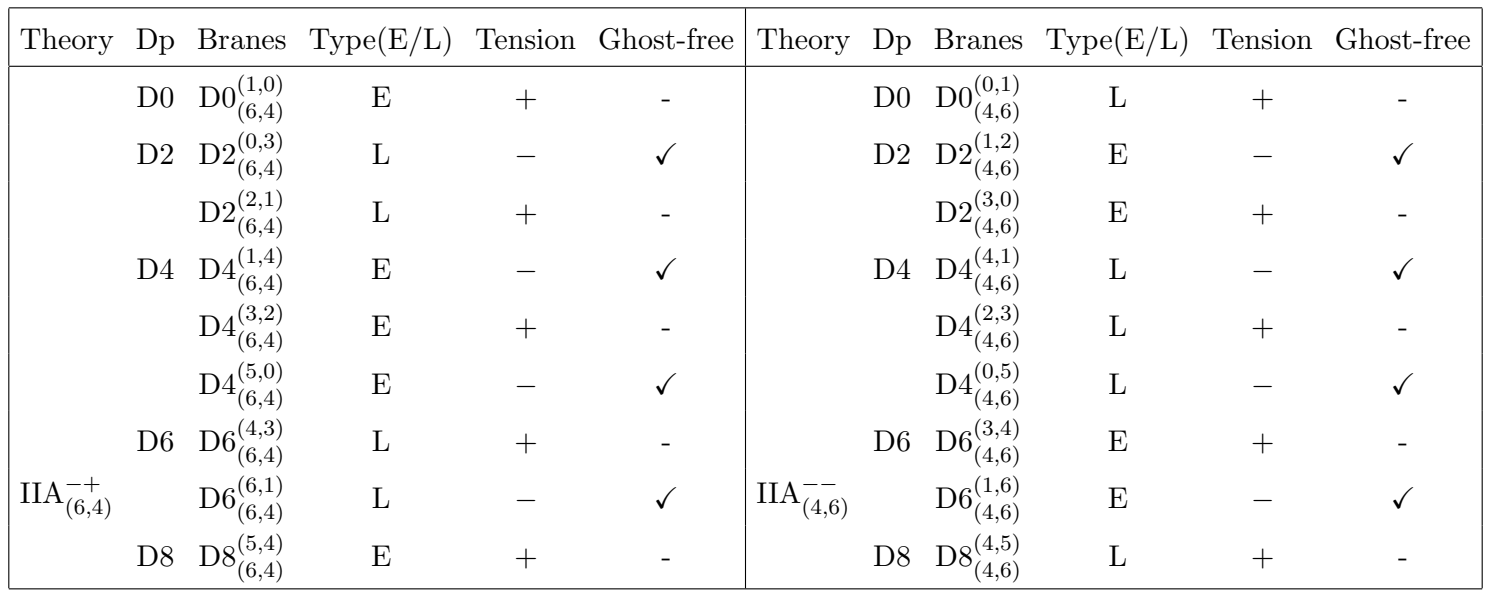

Table 13. Brane spectrum of mirror $\operatorname{IIA}_{(6,4)}^{-+}$and $\operatorname{IIA}_{(4,6)}^{--}$theories.

Open Access. This article is distributed under the terms of the Creative Commons Attribution License (CC-BY 4.0), which permits any use, distribution and reproduction in any medium, provided the original author(s) and source are credited.

\section{References}

[1] C. Vafa, The String landscape and the swampland, hep-th/0509212 [INSPIRE].

[2] E. Palti, The Swampland: Introduction and Review, Fortsch. Phys. 67 (2019) 1900037 [arXiv: 1903.06239] [INSPIRE].

[3] N. Arkani-Hamed, L. Motl, A. Nicolis and C. Vafa, The String landscape, black holes and gravity as the weakest force, JHEP 06 (2007) 060 [hep-th/0601001] [INSPIRE].

[4] H. Ooguri and C. Vafa, On the Geometry of the String Landscape and the Swampland, Nucl. Phys. B 766 (2007) 21 [hep-th/0605264] [INSPIRE].

[5] D. Klaewer and E. Palti, Super-Planckian Spatial Field Variations and Quantum Gravity, JHEP 01 (2017) 088 [arXiv:1610.00010] [InSPIRE].

[6] H. Ooguri and C. Vafa, Non-supersymmetric AdS and the Swampland, Adv. Theor. Math. Phys. 21 (2017) 1787 [arXiv:1610.01533] [INSPIRE]. 
[7] E. Palti, The Weak Gravity Conjecture and Scalar Fields, JHEP 08 (2017) 034 [arXiv: 1705.04328] [INSPIRE].

[8] G. Obied, H. Ooguri, L. Spodyneiko and C. Vafa, de Sitter Space and the Swampland, arXiv: 1806.08362 [INSPIRE].

[9] D. Andriot, On the de Sitter swampland criterion, Phys. Lett. B 785 (2018) 570 [arXiv: 1806.10999] [INSPIRE].

[10] S. Cecotti and C. Vafa, Theta-problem and the String Swampland, arXiv:1808.03483 [INSPIRE].

[11] S.K. Garg and C. Krishnan, Bounds on Slow Roll and the de Sitter Swampland, JHEP 11 (2019) 075 [arXiv: 1807.05193] [INSPIRE].

[12] H. Ooguri, E. Palti, G. Shiu and C. Vafa, Distance and de Sitter Conjectures on the Swampland, Phys. Lett. B 788 (2019) 180 [arXiv:1810.05506] [InSPIRE].

[13] F.F. Gautason, V. Van Hemelryck and T. Van Riet, The Tension between 10D Supergravity and dS Uplifts, Fortsch. Phys. 67 (2019) 1800091 [arXiv:1810.08518] [INSPIRE].

[14] D. Klaewer, D. Lüst and E. Palti, A Spin-2 Conjecture on the Swampland, Fortsch. Phys. 67 (2019) 1800102 [arXiv: 1811.07908] [INSPIRE].

[15] J.J. Heckman and C. Vafa, Fine Tuning, Sequestering and the Swampland, Phys. Lett. B 798 (2019) 135004 [arXiv: 1905.06342] [INSPIRE].

[16] D. Lüst, E. Palti and C. Vafa, AdS and the Swampland, Phys. Lett. B 797 (2019) 134867 [arXiv: 1906.05225] [INSPIRE].

[17] A. Bedroya and C. Vafa, Trans-Planckian Censorship and the Swampland, arXiv: 1909.11063 [INSPIRE].

[18] A. Kehagias, D. Lüst and S. Lüst, Swampland, Gradient Flow and Infinite Distance, JHEP 04 (2020) 170 [arXiv: 1910.00453] [INSPIRE].

[19] R. Blumenhagen, M. Brinkmann and A. Makridou, Quantum Log-Corrections to Swampland Conjectures, JHEP 02 (2020) 064 [arXiv:1910.10185] [INSPIRE].

[20] B. Heidenreich, M. Reece and T. Rudelius, The Weak Gravity Conjecture and Emergence from an Ultraviolet Cutoff, Eur. Phys. J. C 78 (2018) 337 [arXiv:1712.01868] [INSPIRE].

[21] T.W. Grimm, E. Palti and I. Valenzuela, Infinite Distances in Field Space and Massless Towers of States, JHEP 08 (2018) 143 [arXiv: 1802.08264] [INSPIRE].

[22] B. Heidenreich, M. Reece and T. Rudelius, Emergence of Weak Coupling at Large Distance in Quantum Gravity, Phys. Rev. Lett. 121 (2018) 051601 [arXiv:1802.08698] [INSPIRE].

[23] S.-J. Lee, W. Lerche and T. Weigand, Tensionless Strings and the Weak Gravity Conjecture, JHEP 10 (2018) 164 [arXiv: 1808.05958] [INSPIRE].

[24] S.-J. Lee, W. Lerche and T. Weigand, A Stringy Test of the Scalar Weak Gravity Conjecture, Nucl. Phys. B 938 (2019) 321 [arXiv:1810.05169] [InSPIRE].

[25] S.-J. Lee, W. Lerche and T. Weigand, Emergent Strings from Infinite Distance Limits, arXiv: 1910.01135 [INSPIRE].

[26] U.H. Danielsson and T. Van Riet, What if string theory has no de Sitter vacua?, Int. J. Mod. Phys. D 27 (2018) 1830007 [arXiv:1804.01120] [INSPIRE]. 
[27] G. Dvali and C. Gomez, Quantum Exclusion of Positive Cosmological Constant?, Annalen Phys. 528 (2016) 68 [arXiv: 1412.8077] [INSPIRE].

[28] G. Dvali, C. Gomez and S. Zell, Quantum Break-Time of de Sitter, JCAP 06 (2017) 028 [arXiv: 1701.08776] [INSPIRE].

[29] G. Dvali, C. Gomez and S. Zell, Quantum Breaking Bound on de Sitter and Swampland, Fortsch. Phys. 67 (2019) 1800094 [arXiv:1810.11002] [INSPIRE].

[30] G. Dvali and C. Gomez, On Exclusion of Positive Cosmological Constant, Fortsch. Phys. 67 (2019) 1800092 [arXiv: 1806.10877] [INSPIRE].

[31] K. Dasgupta, M. Emelin, M.M. Faruk and R. Tatar, de Sitter Vacua in the String Landscape, arXiv: 1908.05288 [INSPIRE].

[32] K. Dasgupta, M. Emelin, M.M. Faruk and R. Tatar, How a four-dimensional de Sitter solution remains outside the swampland, arXiv:1911.02604 [INSPIRE].

[33] C.M. Hull, Timelike $T$ duality, de Sitter space, large $N$ gauge theories and topological field theory, JHEP 07 (1998) 021 [hep-th/9806146] [INSPIRE].

[34] C.M. Hull, Duality and the signature of space-time, JHEP 11 (1998) 017 [hep-th/9807127] [INSPIRE].

[35] C.M. Hull and R.R. Khuri, Branes, times and dualities, Nucl. Phys. B 536 (1998) 219 [hep-th/9808069] [INSPIRE].

[36] C.M. Hull and R.R. Khuri, World volume theories, holography, duality and time, Nucl. Phys. B 575 (2000) 231 [hep-th/9911082] [INSPIRE].

[37] N. Bobev, P. Bomans and F.F. Gautason, Spherical Branes, JHEP 08 (2018) 029 [arXiv: 1805. 05338] [INSPIRE].

[38] N. Bobev, P. Bomans, F.F. Gautason, J.A. Minahan and A. Nedelin, Supersymmetric Yang-Mills, Spherical Branes and Precision Holography, JHEP 03 (2020) 047 [arXiv: 1910.08555] [INSPIRE].

[39] R. Dijkgraaf, B. Heidenreich, P. Jefferson and C. Vafa, Negative Branes, Supergroups and the Signature of Spacetime, JHEP 02 (2018) 050 [arXiv: 1603. 05665] [INSPIRE].

[40] M.P. Hertzberg, S. Kachru, W. Taylor and M. Tegmark, Inflationary Constraints on Type IIA String Theory, JHEP 12 (2007) 095 [arXiv:0711.2512] [inSPIRE].

[41] H. Ooguri and C. Vafa, $N=2$ heterotic strings, Nucl. Phys. B 367 (1991) 83 [InSPIRE].

[42] J.M. Maldacena and C. Núñez, Supergravity description of field theories on curved manifolds and a no go theorem, Int. J. Mod. Phys. A 16 (2001) 822 [hep-th/0007018] [INSPIRE].

[43] D. Junghans, Weakly Coupled de Sitter Vacua with Fluxes and the Swampland, JHEP 03 (2019) 150 [arXiv: 1811.06990] [INSPIRE].

[44] H. Ooguri and C. Vafa, Geometry of $N=2$ strings, Nucl. Phys. B 361 (1991) 469 [InSPIRE].

[45] I. Quiros, Time-like versus space-like extra dimensions, arXiv:0707.0714 [INSPIRE].

[46] P.A.M. Dirac, Unitary Representations of the Lorentz Group, Proc. Roy. Soc. Lond. A 183 (1945) 284 [INSPIRE].

[47] W. Pauli and F. Villars, On the Invariant regularization in relativistic quantum theory, Rev. Mod. Phys. 21 (1949) 434 [INSPIRE]. 
[48] S.N. Gupta, Theory of longitudinal photons in quantum electrodynamics, Proc. Phys. Soc. A 63 (1950) 681 [INSPIRE].

[49] K. BlEuler, A New method of treatment of the longitudinal and scalar photons, Helv. Phys. Acta 23 (1950) 567 [inSPIRE].

[50] P. Di Vecchia and A. Liccardo, D Branes in String Theory, I, NATO Sci. Ser. C 556 (2000) 1 [hep-th/9912161] [INSPIRE].

[51] P. Di Vecchia and A. Liccardo, D-branes in string theory. 2., in YITP Workshop on Developments in Superstring and M-theory, Kyoto Japan (1999), pg. 7 [hep-th/9912275] [INSPIRE].

[52] R. Blumenhagen and E. Plauschinn, Introduction to conformal field theory, Lect. Notes Phys. 779 (2009) 1 [INSPIRE].

[53] A. Recknagel and V. Schomerus, Boundary Conformal Field Theory and the Worldsheet Approach to D-Branes, Cambridge Monographs on Mathematical Physics, Cambridge University Press, Cambridge U.K. (2013).

[54] C. Angelantonj and A. Sagnotti, Open strings, Phys. Rept. 371 (2002) 1 [Erratum ibid. 376 (2003) 407] [hep-th/0204089] [INSPIRE].

[55] V. Cortés eds., Handbook of Pseudo-Riemannian Geometry and Supersymmetry, IRMA Lectures in Mathematics and Theoretical Physics. Vol. 16, European Mathematical Society, Zürich Switzerland (2010). 